\title{
General and Efficient Indole Syntheses Based on Catalytic Amination Reactions
}

\author{
Lutz Ackermann* \\ Department of Chemistry, Ludwig-Maximilians-Universität München, Butenandtstrasse 5-13, D-81377 \\ München, Germany \\ Lutz.Ackermann@cup.uni-muenchen.de
}

\section{Supporting Information}

\section{General remarks}

All reactions were carried out on a 1 mmol scale under $\mathrm{N}_{2}$ using pre-dried glassware. Chemicals were obtained from commercial sources, and were used without further purification. Toluene was freshly distilled from sodium under nitrogen. Yields refer to isolated compounds, estimated to be $>95 \%$ pure as determined by ${ }^{1} \mathrm{H}-\mathrm{NMR}$ and GC. Flash chromatography: Macherey-Nagel silica gel 60 (70-230 mesh). NMR: Spectra were recorded on Bruker ARX 300 in the solvent indicated; chemical shifts $(\delta)$ are given in ppm, coupling constants $(J)$ in Hz. IR: Wavenumbers in $\mathrm{cm}^{-1}$.

Representative Procedure A for palladium-catalyzed indole synthesis:

\section{2-Phenyl-1-p-tolyl-1H-indole (4a):}

To a solution of $\mathrm{Pd}(\mathrm{OAc})_{2}(11 \mathrm{mg}, 0.05 \mathrm{mmol}, 5 \mathrm{~mol} \%), \mathrm{HIPrCl}(5)$ (21 mg, $0.05 \mathrm{mmol}, 5$ $\mathrm{mol} \%$ ) and $\mathrm{KO} t$-Bu (336 $\mathrm{mg}, 3.0 \mathrm{mmol})$ in $\mathrm{PhMe}(3 \mathrm{~mL})$ was added 1-chloro-2phenylethynyl-benzene $(212 \mathrm{mg}, 1.0 \mathrm{mmol})$ and 4-methylaniline $(129 \mathrm{mg}, 1.2 \mathrm{mmol})$ at room temperature. The resulting red mixture was stirred at $105{ }^{\circ} \mathrm{C}$ for $2 \mathrm{~h}$, after which $\mathrm{GC} / \mathrm{MS}$ analysis indicated complete conversion of the starting material. $\mathrm{CH}_{2} \mathrm{Cl}_{2}(50 \mathrm{~mL})$ and aq. $\mathrm{HCl}$ $(2 \mathrm{~N}, 50 \mathrm{~mL})$ were added to the cooled reaction mixture. The separated aqueous phase was extracted with $\mathrm{CH}_{2} \mathrm{Cl}_{2}(2 \times 50 \mathrm{~mL})$. The combined organic layers were dried over $\mathrm{MgSO}_{4}$ and 
concentrated in vacuo. The remaining residue was purified by column chromatography on silica gel (n-pentane/Et $\left.{ }_{2} \mathrm{O}, 200 / 1\right)$ to yield 4a as a white solid (282 mg, $99 \%$ ).

\section{Representative Procedure B for CuI-catalyzed indole synthesis:}

\section{2-n-Hexyl-1-p-tolyl-1H-indole (4n):}

To a solution of $\mathrm{CuI}(18 \mathrm{mg}, 0.10 \mathrm{mmol}, 10 \mathrm{~mol} \%)$ and $\mathrm{KO} t$-Bu (336 mg, $3.0 \mathrm{mmol})$ in $\mathrm{PhMe}$ (3 mL) was added 1-bromo-2-oct-1-ynyl-benzene $(265 \mathrm{mg}, 1.0 \mathrm{mmol})$ and 4-methylaniline (129 mg, $1.2 \mathrm{mmol}$ ) at room temperature. The resulting mixture was stirred at $105^{\circ} \mathrm{C}$ for $2 \mathrm{~h}$, after which GC/MS analysis indicated complete conversion of the starting material. $\mathrm{CH}_{2} \mathrm{Cl}_{2}$ $(50 \mathrm{~mL})$ and aq. $\mathrm{HCl}(2 \mathrm{~N}, 50 \mathrm{~mL})$ were added to the cooled reaction mixture. The separated aqueous phase was extracted with $\mathrm{CH}_{2} \mathrm{Cl}_{2}(2 \times 50 \mathrm{~mL})$. The combined organic layers were dried over $\mathrm{MgSO}_{4}$ and concentrated in vacuo. The remaining residue was purified by column chromatography on silica gel $(n$-pentane/Et $2 \mathrm{O}, 200 / 1 \rightarrow 50 / 1)$ to yield $\mathbf{4 n}$ as a yellow oil (195 $\mathrm{mg}, 67 \%)$.

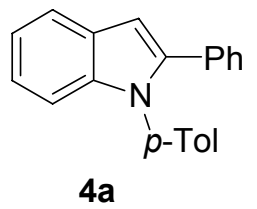

\section{2-Phenyl-1-p-tolyl-1 $H$-indole (4a):}

${ }^{1} \mathrm{H}$ NMR $\left(300 \mathrm{MHz}, \mathrm{CDCl}_{3}\right): \delta=7.59(\mathrm{dd}, J=6.2,2.9 \mathrm{~Hz}, 1 \mathrm{H}), 7.22-7.00(\mathrm{~m}, 12 \mathrm{H}), 6.70(\mathrm{~d}$, $J=0.7 \mathrm{~Hz}, 1 \mathrm{H}), 2.31(\mathrm{~s}, 3 \mathrm{H}) .{ }^{13} \mathrm{C}-\mathrm{NMR}\left(75 \mathrm{MHz}, \mathrm{CDCl}_{3}\right): \delta=140.8,139.1,137.0,135.9$, 132.6, 129.9, 128.9, 128.2, 128.1, 127.8, 127.2, 122.2, 120.5, 120.4, 110.7, 103.4, 21.1. IR (KBr): 3029, 1511, 1456, 1440, 1351, 1320, 1209, 1109, 793, 761, 747, $697 \mathrm{~cm}^{-1}$. MS (EI) $m / z$ (relative intensity) $283(100)\left[\mathrm{M}^{+}\right], 282$ (12), 267 (10), 165 (6). HR-MS (EI) $\mathrm{m} / z$ calcd for $\mathrm{C}_{21} \mathrm{H}_{17} \mathrm{~N} 283,1361$, found 283,1375. 


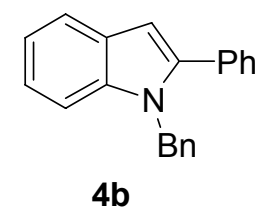

\section{1-Benzyl-2-phenyl-1H-indole (4b):}

Following general procedure A, indole $4 \mathbf{b}(262 \mathrm{mg}, 92 \%)$ was obtained as a white solid after purification by column chromatography on silica gel (n-pentane/ $\left.\mathrm{Et}_{2} \mathrm{O}, 200 / 1\right) .{ }^{1} \mathrm{H}$ NMR (300 $\left.\mathrm{MHz}, \mathrm{CDCl}_{3}\right): \delta=7.58(\mathrm{dd}, J=5.9,0.8 \mathrm{~Hz}, 1 \mathrm{H}), 7.36-7.00(\mathrm{~m}, 11 \mathrm{H}), 6.92(\mathrm{dd}, J=8.2,1.8$ $\mathrm{Hz}, 2 \mathrm{H}), 6.56(\mathrm{~s}, 1 \mathrm{H}), 5.26(\mathrm{~s}, 2 \mathrm{H}) .{ }^{13} \mathrm{C}-\mathrm{NMR}\left(75 \mathrm{MHz}, \mathrm{CDCl}_{3}\right): \delta=141.8,138.2,138.0$, $132.7,129.2,128.7,128.5,128.3,128.0,127.1,125.9,121.9,120.5,120.1,110.5,102.3,47.7$. IR (KBr): 3026, 1494, 1462, 1344, 1306, 1175, 1158, 1026, 772, 749, $697 \mathrm{~cm}^{-1}$. MS (EI) $\mathrm{m} / z$ (relative intensity) $283(100)\left[\mathrm{M}^{+}\right], 193$ (10), 165 (11), 91 (77). HR-MS (EI) $\mathrm{m} / z$ calcd for $\mathrm{C}_{21} \mathrm{H}_{17} \mathrm{~N} 283,1361$, found 283,1376.

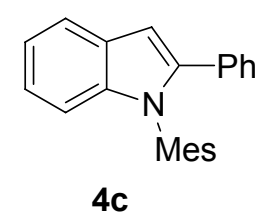

\section{2-Phenyl-1-(2,4,6-trimethylphenyl)-1H-indole (4c):}

Following general procedure A, indole 4c (309 mg, 99\%) was obtained as a white solid after purification by column chromatography on silica gel (n-pentane/Et $\left.{ }_{2} \mathrm{O}, 200 / 1 \rightarrow 100 / 1\right) .{ }^{1} \mathrm{H}$ NMR (300 MHz, $\left.\mathrm{CDCl}_{3}\right): \delta=7.59(\mathrm{~m}, 1 \mathrm{H}), 7.20-7.00(\mathrm{~m}, 7 \mathrm{H}), 6.87(\mathrm{~s}, 2 \mathrm{H}), 6.76(\mathrm{~s}, 1 \mathrm{H})$, $6.71(\mathrm{~m}, 1 \mathrm{H}), 2.23(\mathrm{~s}, 3 \mathrm{H}), 1.72(\mathrm{~s}, 6 \mathrm{H}) .{ }^{13} \mathrm{C}-\mathrm{NMR}\left(75 \mathrm{MHz}, \mathrm{CDCl}_{3}\right): \delta=140.5,138.0,138.0$ $137.9,136.9,133.9,132.8,129.2,128.3,127.5,127.2,122.1,120.4,120.2,110.5,102.0,21.1$, 17.7. IR (KBr): 3057, 3027, 2919, 1603, 1472, 1375, 1210, 1029, 855, 791, 738, $695 \mathrm{~cm}^{-1}$. MS (EI) $m / z$ (relative intensity) 311 (100) $\left[\mathrm{M}^{+}\right], 296$ (19), 234 (13), 140 (12). HR-MS (EI) $m / z$ calcd for $\mathrm{C}_{23} \mathrm{H}_{21} \mathrm{~N} 311.1674$, found 311.1698. 


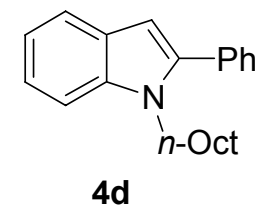

\section{1-n-Octyl-2-phenyl-1H-indole (4d):}

Following general procedure A, indole 4d (284 mg, 93\%) was obtained as a white solid after purification by column chromatography on silica gel (n-pentane/ $\left.\mathrm{Et}_{2} \mathrm{O}, 200 / 1\right) .{ }^{1} \mathrm{H}$ NMR (300 $\left.\mathrm{MHz}, \mathrm{CDCl}_{3}\right): \delta=7.54(\mathrm{~d}, J=7.9 \mathrm{~Hz}, 1 \mathrm{H}), 7.43-7.20(\mathrm{~m}, 6 \mathrm{H}), 7.14(\mathrm{dt}, J=7.6,1.3 \mathrm{~Hz}, 1 \mathrm{H})$, $7.04(\mathrm{dt}, J=7.4,1.0 \mathrm{~Hz}, 1 \mathrm{H}), 6.43(\mathrm{~d}, J=0.7 \mathrm{~Hz}, 1 \mathrm{H}), 4.05(\mathrm{t}, J=7.6 \mathrm{~Hz}, 2 \mathrm{H}), 1.60(\mathrm{t}, J=$ $7.4 \mathrm{~Hz}, 2 \mathrm{H}), 1.20-1.05(\mathrm{~m}, 10 \mathrm{H}), 0.77(\mathrm{t}, J=7.0 \mathrm{~Hz}, 3 \mathrm{H}) .{ }^{13} \mathrm{C}-\mathrm{NMR}\left(75 \mathrm{MHz}, \mathrm{CDCl}_{3}\right): \delta=$ $141.3,137.3,133.3,129.4,128.4,128.2,127.9,121.4,120.5,119.7,110.0,102.0,43.9,31.7$, 29.9, 29.1, 29.0, 26.7, 22.6, 14.1. IR (KBr): 3030, 2927, 2854, 1605, 1460, 1348, 1161, 1013, 786, 748, $699 \mathrm{~cm}^{-1}$. MS (EI) $\mathrm{m} / z$ (relative intensity) $305(70)\left[\mathrm{M}^{+}\right], 207$ (18), $206(100), 204$ (12). HR-MS (EI) $m / z$ calcd for $\mathrm{C}_{22} \mathrm{H}_{27} \mathrm{~N} 305.2143$, found 305.2129 .

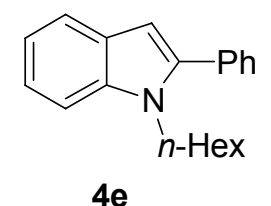

\section{1-n-Hexyl-2-phenyl-1H-indole (4e):}

Following general procedure A, indole 4 e $(258 \mathrm{mg}$, 93\%) was obtained as a yellow oil after purification by column chromatography on silica gel (n-pentane/Et $2 \mathrm{O}, 100 / 1) .{ }^{1} \mathrm{H}$ NMR (300 $\left.\mathrm{MHz}, \mathrm{CDCl}_{3}\right): \delta=7.54(\mathrm{~d}, J=7.9 \mathrm{~Hz}, 1 \mathrm{H}), 7.41-7.25(\mathrm{~m}, 6 \mathrm{H}), 7.13(\mathrm{ddd}, J=7.9,7.0,1.2 \mathrm{~Hz}$ 1H), 7.03 (ddd, $J=7.6,7.0,1.1 \mathrm{~Hz}, 1 \mathrm{H}), 6.42(\mathrm{~s}, 1 \mathrm{H}), 4.04$ (t, $J=7.6 \mathrm{~Hz}, 2 \mathrm{H}), 1.59$ (quint., $J$ $=7.4 \mathrm{~Hz}, 2 \mathrm{H}), 1.15-1.00(\mathrm{~m}, 6 \mathrm{H}), 0.72(\mathrm{t}, J=6.7 \mathrm{~Hz}, 3 \mathrm{H}) .{ }^{13} \mathrm{C}-\mathrm{NMR}\left(75 \mathrm{MHz}, \mathrm{CDCl}_{3}\right): \delta=$ $141.3,137.3,133.3,129.4,128.4,128.2,127.8,121.4,120.5,119.7,110.0,102.0,43.9,31.2$, 29.9, 26.4, 22.4, 13.9. IR (KBr): 3058, 2955, 2857, 1605, 1461, 1348, 1162, 1013, 786, 748, $699 \mathrm{~cm}^{-1}$. MS (EI) m/z (relative intensity) 277 (59) $\left[\mathrm{M}^{+}\right], 207$ (19), 206 (100), 204 (20). HRMS (EI) $m / z$ calcd for $\mathrm{C}_{20} \mathrm{H}_{23} \mathrm{~N} 277.1830$, found 277.1851 . 


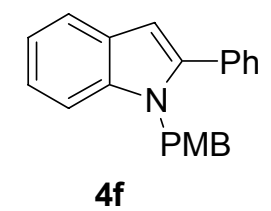

\section{1-(p-Methoxybenzyl)-2-phenyl-1H-indole (4f):}

Following general procedure A, indole $4 \mathbf{f}(246 \mathrm{mg}$, 78\%) was obtained as a white solid after purification by column chromatography on silica gel ( $n$-pentane/ $\left.\mathrm{Et}_{2} \mathrm{O}, 100 / 1 \rightarrow 50 / 1\right) .{ }^{1} \mathrm{H} \mathrm{NMR}$ $\left(300 \mathrm{MHz}, \mathrm{CDCl}_{3}\right): \delta=7.57(\mathrm{~m}, 1 \mathrm{H}), 7.40-7.25(\mathrm{~m}, 5 \mathrm{H}), 7.15-7.00(\mathrm{~m}, 3 \mathrm{H}), 6.85(\mathrm{~d}, J=8.7$ $\mathrm{Hz}, 2 \mathrm{H}), 6.71(\mathrm{~d}, J=8.7 \mathrm{~Hz}, 2 \mathrm{H}), 6.55(\mathrm{~s}, 1 \mathrm{H}), 5.22(\mathrm{~s}, 2 \mathrm{H}), 3.67(\mathrm{~s}, 3 \mathrm{H}) .{ }^{13} \mathrm{C}-\mathrm{NMR}(75 \mathrm{MHz}$, $\left.\mathrm{CDCl}_{3}\right): \delta=158.7,141.7,137.9,132.8,130.2,129.2,128.5,128.3,128.0,127.1,121.8$ 120.5, 120.1, 114.1, 110.6, 102.3, 55.2, 47.2. IR (KBr): 3058, 2956, 2830, 1614, 1510, 1463, 1292, 1176, 1036, 820, 754, $704 \mathrm{~cm}^{-1}$. MS (EI) $\mathrm{m} / z$ (relative intensity) $313(32)\left[\mathrm{M}^{+}\right], 193$ (12), 165 (6), 121 (100). HR-MS (EI) $m / z$ calcd for $\mathrm{C}_{22} \mathrm{H}_{19} \mathrm{NO} 313.1467$, found 313.1441.

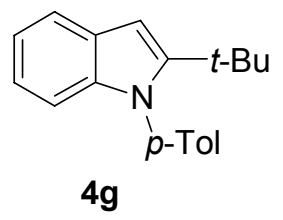

\section{2-t-Butyl-1-p-tolyl-1H-indole (4g):}

Following general procedure A, indole $4 \mathrm{~g}(218 \mathrm{mg}, 83 \%)$ was obtained as a white solid after purification by column chromatography on silica gel (n-pentane/Et $\left.{ }_{2} \mathrm{O}, 200 / 1\right) .{ }^{1} \mathrm{H}$ NMR (300 $\left.\mathrm{MHz}, \mathrm{CDCl}_{3}\right): \delta=7.47(\mathrm{dd}, J=7.8,1.1 \mathrm{~Hz}, 1 \mathrm{H}), 7.25-7.10(\mathrm{~m}, 4 \mathrm{H}), 6.98(\mathrm{dt}, J=7.1,1.2 \mathrm{~Hz}$ 1H), $6.92(\mathrm{dt}, J=7.1,1.4 \mathrm{~Hz}, 1 \mathrm{H}), 6.58(\mathrm{~d}, J=8.0 \mathrm{~Hz}, 1 \mathrm{H}), 6.37(\mathrm{~d}, J=0.7 \mathrm{~Hz}, 1 \mathrm{H}), 2.38(\mathrm{~s}$, 3H), $1.18(\mathrm{~s}, 9 \mathrm{H}) .{ }^{13} \mathrm{C}-\mathrm{NMR}\left(75 \mathrm{MHz}, \mathrm{CDCl}_{3}\right): \delta=150.7,140.9,138.4,137.5,130.4,129.6$, 126.8, 121.0, 119.7, 119.5, 110.2, 99.0, 33.2, 31.0, 21.2. IR (KBr): 3054, 2967, 2867, 1511, 1456, 1358, 1255, 1014, 822, $780 \mathrm{~cm}^{-1}$. MS (EI) $\mathrm{m} / z$ (relative intensity) $263(72)\left[\mathrm{M}^{+}\right], 248$ (100), 233 (14), 218 (27). HR-MS (EI) $m / z$ calcd for $\mathrm{C}_{19} \mathrm{H}_{21} \mathrm{~N} 263.1674$, found 263.1693. 


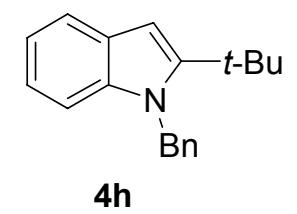

\section{1-Benzyl-2-t-butyl-1H-indole (4h):}

Following general procedure A, indole $4 \mathbf{h}$ (195 mg, 74\%) was obtained as a pale-yellow solid after purification by column chromatography on silica gel (n-pentane/Et $2 \mathrm{O}, 200 / 1) .{ }^{1} \mathrm{H}$ NMR $\left(300 \mathrm{MHz}, \mathrm{CDCl}_{3}\right): \delta=7.57(\mathrm{~m}, 1 \mathrm{H}), 7.27-7.15(\mathrm{~m}, 3 \mathrm{H}), 7.10-6.95(\mathrm{~m}, 3 \mathrm{H}), 6.87(\mathrm{~d}, J=6.8$ $\mathrm{Hz}, 2 \mathrm{H}), 6.41(\mathrm{~s}, 1 \mathrm{H}), 5.60(\mathrm{~s}, 2 \mathrm{H}), 1.42(\mathrm{~s}, 9 \mathrm{H}) .{ }^{13} \mathrm{C}-\mathrm{NMR}\left(75 \mathrm{MHz}, \mathrm{CDCl}_{3}\right): \delta=149.2$, $138.2,138.2,128.6,127.5,126.9,125.6,121.1,120.0,119.6,109.9,98.7,48.5,32.4,30.6$. IR (KBr): 3031, 2968, 1528, 1466, 1345, 1310, 1245, 786, $728 \mathrm{~cm}^{-1}$. MS (EI) $\mathrm{m} / z$ (relative intensity) $263(80)\left[\mathrm{M}^{+}\right], 249$ (20), 248 (98), 91 (100). HR-MS (EI) $\mathrm{m} / z$ calcd for $\mathrm{C}_{19} \mathrm{H}_{21} \mathrm{~N}$ 263.1674, found 263.1670 .<smiles>FC(F)(F)c1ccc2c(c1)cc(C=Cc1ccccc1)n2Br</smiles>

\section{1-Benzyl-2-n-hexyl-5-trifluoromethyl-1H-indole (4i):}

Following general procedure A, indole $4 \mathbf{i}(238 \mathrm{mg}, 66 \%)$ was obtained as a pale-yellow oil after purification by column chromatography on silica gel (n-pentane/Et $\left.{ }_{2} \mathrm{O}, 200 / 1\right) .{ }^{1} \mathrm{H} \mathrm{NMR}$ $\left(300 \mathrm{MHz}, \mathrm{CDCl}_{3}\right): \delta=7.78(\mathrm{~s}, 1 \mathrm{H}), 7.25-7.10(\mathrm{~m}, 5 \mathrm{H}), 6.84(\mathrm{dd}, J=7.5,2.0 \mathrm{~Hz}, 2 \mathrm{H}), 6.34$ $(\mathrm{d}, J=0.5 \mathrm{~Hz}, 1 \mathrm{H}), 5.25(\mathrm{~s}, 2 \mathrm{H}), 2.59(\mathrm{t}, J=7.6 \mathrm{~Hz}, 2 \mathrm{H}), 1.61$ (quint., $J=7.5 \mathrm{~Hz}, 2 \mathrm{H}), 1.32-$ $1.10(\mathrm{~m}, 6 \mathrm{H}), 0.79(\mathrm{t}, J=6.8 \mathrm{~Hz}, 3 \mathrm{H}) .{ }^{13} \mathrm{C}-\mathrm{NMR}\left(75 \mathrm{MHz}, \mathrm{CDCl}_{3}\right): \delta=143.4,138.5,137.3$, $128.8,127.6,127.5,125.8,125.5\left(\mathrm{q},{ }^{1} J=271 \mathrm{~Hz}\right), 122.3\left(\mathrm{q},{ }^{2} J=32 \mathrm{~Hz}\right), 117.6\left(\mathrm{q},{ }^{3} J=4 \mathrm{~Hz}\right)$, $117.4\left(\mathrm{q},{ }^{3} J=4 \mathrm{~Hz}\right), 109.4,100.3,46.6,31.2,29.0,28.2,26.7,22.5,14.0 . \mathrm{IR}(\mathrm{KBr}): 3032$, 2956, 2930, 2859, 1621, 1551, 1453, 1330, 1273, 1160, 1112, 1057, 889, 803, 726, $696 \mathrm{~cm}^{-1}$. MS (EI) $m / z$ (relative intensity) 359 (25) $\left[\mathrm{M}^{+}\right], 289$ (60), 288 (34), 91 (100). HR-MS (EI) $m / z$ calcd for $\mathrm{C}_{22} \mathrm{H}_{24} \mathrm{NF}_{3} 359.1861$, found 359.1844. 
<smiles>C=Cc1cc2cc(C(F)(F)F)ccc2n1-c1ccc(C(=O)OCC)cc1</smiles>

$4 \mathbf{j}$

\section{1-(p-Ethoxycarbonylphenyl)-2-n-hexyl-5-trifluoromethyl- $1 H$-indole $(4 \mathrm{j})$ :}

Following general procedure A, indole $4 \mathbf{j}(279 \mathrm{mg}, 67 \%)$ was obtained as a pale-yellow oil after purification by column chromatography on silica gel (n-pentane/ $\mathrm{Et}_{2} \mathrm{O}$, $200 / 1 \rightarrow 100 / 1 \rightarrow 50 / 1 \rightarrow 25 / 1) .{ }^{1} \mathrm{H}$ NMR $\left(300 \mathrm{MHz}, \mathrm{CDCl}_{3}\right): \delta=8.26(\mathrm{~d}, J=8.6 \mathrm{~Hz}, 2 \mathrm{H}), 7.88$ (s, 1H), $7.43(\mathrm{~d}, J=8.6 \mathrm{~Hz}, 2 \mathrm{H}), 7.33(\mathrm{dd}, J=8.6,1.6 \mathrm{~Hz}, 1 \mathrm{H}), 7.13(\mathrm{~d}, J=8.6 \mathrm{~Hz}, 1 \mathrm{H}), 6.53$ $(\mathrm{d}, J=0.8 \mathrm{~Hz}, 1 \mathrm{H}), 4.46(\mathrm{q}, J=7.1 \mathrm{~Hz}, 2 \mathrm{H}), 2.64(\mathrm{t}, J=7.5 \mathrm{~Hz}, 2 \mathrm{H}), 1.58$ (quint., $J=7.4 \mathrm{~Hz}$, 2H), $1.46(\mathrm{t}, J=7.1 \mathrm{~Hz}, 3 \mathrm{H}), 1.35-1.20(\mathrm{~m}, 6 \mathrm{H}), 0.86(\mathrm{t}, J=6.8 \mathrm{~Hz}, 3 \mathrm{H}) .{ }^{13} \mathrm{C}-\mathrm{NMR}(75 \mathrm{MHz}$, $\left.\mathrm{CDCl}_{3}\right): \delta=165.7,143.6,141.4,139.2,131.0,130.3,127.9,127.7,125.3\left(\mathrm{q},{ }^{1} J=271 \mathrm{~Hz}\right)$, $122.7\left(\mathrm{q},{ }^{2} J=32 \mathrm{~Hz}\right), 118.2\left(\mathrm{q},{ }^{3} J=4 \mathrm{~Hz}\right), 117.5\left(\mathrm{q},{ }^{3} J=4 \mathrm{~Hz}\right), 110.0,101.7,61.4,31.4,28.8$, 28.4, 27.1, 22.4, 14.3, 14.0. IR (KBr): 3068, 2958, 2932, 2860, 1721, 1607, 1554, 1452, 1331, 1276, 1161, 1114, 1057, 890, 808, 773, $706 \mathrm{~cm}^{-1}$. MS (EI) $\mathrm{m} / z$ (relative intensity) 417 (25) $\left[\mathrm{M}^{+}\right], 360$ (25), 347 (100), 272 (14). HR-MS (EI) $m / z$ calcd for $\mathrm{C}_{24} \mathrm{H}_{26} \mathrm{NO}_{2} \mathrm{~F}_{3}$ 417.1916, found 417.1920. 


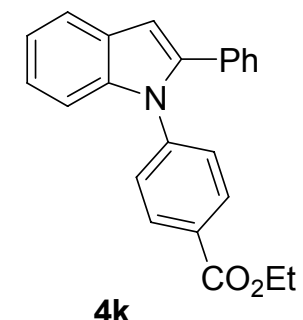

\section{1-(p-Ethoxycarbonylphenyl)-2-phenyl-1H-indole (4k):}

Following general procedure A, indole 4k (313 mg, 92\%) was obtained as a white solid after purification by column chromatography on silica gel ( $n$-pentane/ $\left.\mathrm{Et}_{2} \mathrm{O}, 100 / 1 \rightarrow 50 / 1 \rightarrow 25 / 1\right)$. ${ }^{1} \mathrm{H}$ NMR $\left(300 \mathrm{MHz}, \mathrm{CDCl}_{3}\right): \delta=7.98(\mathrm{~d}, J=8.6 \mathrm{~Hz}, 2 \mathrm{H}), 7.58(\mathrm{ddd}, J=6.1,3.1,0.7 \mathrm{~Hz}$, 1H), $7.23(\mathrm{~m}, 1 \mathrm{H}), 7.19(\mathrm{~d}, J=8.6 \mathrm{~Hz}, 2 \mathrm{H}), 7.16-7.12(\mathrm{~m}, 5 \mathrm{H}), 7.10(\mathrm{~d}, J=6.1 \mathrm{~Hz}, 1 \mathrm{H}), 7.09$ $(\mathrm{d}, J=6.1 \mathrm{~Hz}, 1 \mathrm{H}), 6.71(\mathrm{~d}, J=0.8 \mathrm{~Hz}, 1 \mathrm{H}), 4.29(\mathrm{q}, J=7.1 \mathrm{~Hz}, 2 \mathrm{H}), 1.29(\mathrm{t}, J=7.1 \mathrm{~Hz}, 3 \mathrm{H})$. ${ }^{13} \mathrm{C}-\mathrm{NMR}\left(75 \mathrm{MHz}, \mathrm{CDCl}_{3}\right): \delta=165.9,142.5,140.5,138.5,132.2,130.6,128.9,128.9$, $128.5,128.3,127.5,127.5,122.7,121.1,120.7,110.4,104.8,61.1,14.3 . \mathrm{IR}(\mathrm{KBr}): 3057$, $2978,1716,1602,1512,1456,1383,1275,1170,1112,1020,864,763,745 \mathrm{~cm}^{-1}$. MS (EI) $m / z$ (relative intensity) $341(100)\left[\mathrm{M}^{+}\right], 313$ (32), 267 (14). HR-MS (EI) $\mathrm{m} / \mathrm{z}$ calcd for $\mathrm{C}_{23} \mathrm{H}_{19} \mathrm{NO}_{2}$ 341.1416, found 341.1434. 


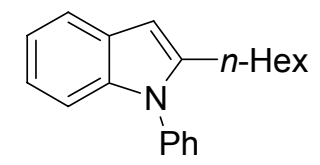

$4 \mathrm{~m}$

\section{2-(n-Hexyl)-1-phenyl-1H-indole (4m):}

Following general procedure A, indole $4 \mathbf{m}$ (233 mg, 84\%) was obtained as a pale-yellow oil after purification by column chromatography on silica gel ( $n$-pentane/Et $2 \mathrm{O}, 200 / 1 \rightarrow 50 / 1) .{ }^{1} \mathrm{H}$ NMR (300 MHz, $\left.\mathrm{CDCl}_{3}\right): \delta=7.65-7.45(\mathrm{~m}, 4 \mathrm{H}), 7.40-7.35(\mathrm{~m}, 2 \mathrm{H}), 7.26-7.00(\mathrm{~m}, 3 \mathrm{H}), 6.46$ (s, 1H), 2.65 (t, $J=7.7 \mathrm{~Hz}, 2 \mathrm{H}), 1.61$ (quint., $J=7.5 \mathrm{~Hz}, 2 \mathrm{H}), 1.40-1.20(\mathrm{~m}, 6 \mathrm{H}), 0.89$ (t, $J=$ $6.8 \mathrm{~Hz}, 3 \mathrm{H}) .{ }^{13} \mathrm{C}-\mathrm{NMR}\left(75 \mathrm{MHz}, \mathrm{CDCl}_{3}\right): \delta=142.0,138.3,138.1,129.4,128.3,128.1,127.8$, 121.0, 119.9, 119.6, 109.9, 100.1, 31.5, 28.9, 28.6, 27.1, 22.5, 14.0. IR (KBr): 3056, 2954, 2856, 1596, 1498, 1456, 1394, 1211, 1016, 778, 762, $699 \mathrm{~cm}^{-1}$. MS (EI) $\mathrm{m} / z$ (relative intensity) 277 (33) $\left[\mathrm{M}^{+}\right], 220$ (26), 207 (100), 206 (90), 204 (26). HR-MS (EI) m/z calcd for $\mathrm{C}_{20} \mathrm{H}_{23} \mathrm{~N}$ 277.1830, found 277.1816.

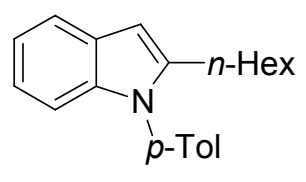

$4 n$

\section{2-(n-Hexyl)-1-p-tolyl-1 $H$-indole (4n):}

Following general procedure A, indole 4n (195 mg, 67\%) was obtained as a pale-yellow oil after purification by column chromatography on silica gel (n-pentane/Et $\left.\mathrm{H}_{2} \mathrm{O}, 200 / 1 \rightarrow 50 / 1\right) .{ }^{1} \mathrm{H}$ $\operatorname{NMR}\left(300 \mathrm{MHz}, \mathrm{CDCl}_{3}\right): \delta=7.60(\mathrm{~m}, 1 \mathrm{H}), 7.35(\mathrm{~d}, J=8.0 \mathrm{~Hz}, 2 \mathrm{H}), 7.25(\mathrm{~d}, J=8.0 \mathrm{~Hz}, 2 \mathrm{H})$, 7.15-7.05 (m, 3H), $6.43(\mathrm{~s}, 1 \mathrm{H}), 2.63(\mathrm{t}, J=7.6 \mathrm{~Hz}, 2 \mathrm{H}), 2.49(\mathrm{~s}, 3 \mathrm{H}), 1.61$ (quint., $J=7.5 \mathrm{~Hz}$, $2 \mathrm{H}), 1.40-1.20(\mathrm{~m}, 6 \mathrm{H}), 0.88(\mathrm{t}, J=6.8 \mathrm{~Hz}, 3 \mathrm{H}) .{ }^{13} \mathrm{C}-\mathrm{NMR}\left(75 \mathrm{MHz}, \mathrm{CDCl}_{3}\right): \delta=142.1$, $138.4,137.6,135.4,130.0,128.0,128.0,120.8,119.8,119.5,110.0,99.8,31.5,28.9,28.6$, 27.0, 22.5, 21.2, 14.0. IR (KBr): 3054, 2954, 2926, 2856, 1608, 1514, 1457, 1394, 1211, 1016, 817, $746 \mathrm{~cm}^{-1}$. MS (EI) $\mathrm{m} / z$ (relative intensity) $291(38)\left[\mathrm{M}^{+}\right], 234$ (28), $221(100), 220$ (65), 204 (29). HR-MS (EI) $m / z$ calcd for $\mathrm{C}_{21} \mathrm{H}_{25} \mathrm{~N} 291.1987$, found 291.1971. 


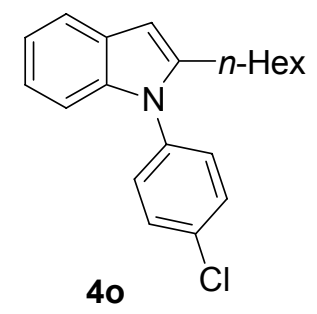

\section{1-(p-Chlorophenyl)-2-(n-hexyl)-1H-indole (4o):}

Following general procedure A, indole $40(210 \mathrm{mg}, 70 \%)$ was obtained as a colorless oil after purification by column chromatography on silica gel (n-pentane/Et $2 \mathrm{O}, 200 / 1) .{ }^{1} \mathrm{H}$ NMR (300 $\left.\mathrm{MHz}, \mathrm{CDCl}_{3}\right): \delta=7.80(\mathrm{~m}, 1 \mathrm{H}), 7.71(\mathrm{~d}, 8.5 \mathrm{~Hz}, 2 \mathrm{H}), 7.49(\mathrm{~d}, J=8.5 \mathrm{~Hz}, 2 \mathrm{H}), 7.36-7.23(\mathrm{~m}$, 3H), $6.64(\mathrm{~s}, 1 \mathrm{H}), 2.81(\mathrm{t}, J=7.6 \mathrm{~Hz}, 2 \mathrm{H}), 1.79$ (quint., $J=7.5 \mathrm{~Hz}, 2 \mathrm{H}), 1.55-1.40(\mathrm{~m}, 6 \mathrm{H})$, $1.08(\mathrm{t}, J=6.8 \mathrm{~Hz}, 3 \mathrm{H}) .{ }^{13} \mathrm{C}-\mathrm{NMR}\left(75 \mathrm{MHz}, \mathrm{CDCl}_{3}\right): \delta=141.7,138.2,136.6,133.6,129.7$, $129.5,128.2,121.2,120.2,119.8,109.7,100.6,31.5,28.9,28.5,27.0,22.5,14.0 . \mathrm{IR}(\mathrm{KBr})$ : $3055,2954,2927,2856,1609,1551,1493,1392,1210,1090,1015,828,746 \mathrm{~cm}^{-1} . \mathrm{MS}(\mathrm{EI})$ $m / z$ (relative intensity) $311(48)\left[\mathrm{M}^{+}\right], 254$ (30), 241 (100), 240 (50), 204 (51). HR-MS (EI) $m / z$ calcd for $\mathrm{C}_{20} \mathrm{H}_{22} \mathrm{NCl} 311.1441$, found 311.1429 .<smiles>CCCc1cc2ccccc2n1[Po]=[Te]</smiles>

\section{2-(n-Butyl)-1-p-tolyl-1H-indole (4p):}

Following general procedure A, indole $4 \mathbf{p}(198 \mathrm{mg}$, 75\%) was obtained as a colorless oil after purification by column chromatography on silica gel (n-pentane/ $\left.\mathrm{Et}_{2} \mathrm{O}, 400 / 1\right) .{ }^{1} \mathrm{H}$ NMR (300 $\left.\mathrm{MHz}, \mathrm{CDCl}_{3}\right): \delta=7.74(\mathrm{md}, J=7.0 \mathrm{~Hz}, 1 \mathrm{H}), 7.48(\mathrm{~d}, 8.2 \mathrm{~Hz}, 2 \mathrm{H}), 7.38(\mathrm{~d}, J=8.2 \mathrm{~Hz}, 2 \mathrm{H})$, 7.30-7.15 (m, 3H), 6.57 (s, 1H), 2.78 (t, $J=7.7 \mathrm{~Hz}, 2 \mathrm{H}), 1.75$ (quint., $J=7.6 \mathrm{~Hz}, 2 \mathrm{H}), 1.49$ (quint., $J=7.4 \mathrm{~Hz}, 2 \mathrm{H}), 1.03(\mathrm{t}, J=7.3 \mathrm{~Hz}, 3 \mathrm{H}) .{ }^{13} \mathrm{C}-\mathrm{NMR}\left(75 \mathrm{MHz}, \mathrm{CDCl}_{3}\right): \delta=142.0$, $138.4,137.6,135.4,130.0,128.0,128.0,120.9,119.8,119.5,110.0,99.8,30.7,26.7,22.3$, 21.2, 13.8. IR (KBr): 3055, 2955, 2928, 2860, 1608, 1548, 1515, 1457, 1394, 1212, 1107 , 
1016, 817, $774 \mathrm{~cm}^{-1}$. MS (EI) $\mathrm{m} / z$ (relative intensity) $263(43)\left[\mathrm{M}^{+}\right], 221$ (100), 220 (79), 205 (32), 204 (37). HR-MS (EI) $m / z$ calcd for $\mathrm{C}_{19} \mathrm{H}_{21} \mathrm{~N} 263.1674$, found 263.1674.

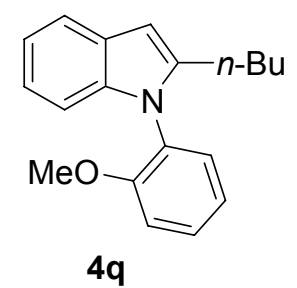

\section{2-(n-Butyl)-1-(o-methoxyphenyl)-1H-indole (4q):}

Following general procedure A, indole 4q (192 mg, 69\%) was obtained as a colorless oil after purification by column chromatography on silica gel (n-pentane/ $\left.\mathrm{Et}_{2} \mathrm{O}, 200 / 1\right) .{ }^{1} \mathrm{H}$ NMR (300 $\left.\mathrm{MHz}, \mathrm{CDCl}_{3}\right): \delta=7.62(\mathrm{dd}, J=5.9,1.8 \mathrm{~Hz}, 1 \mathrm{H}), 7.48(\mathrm{ddd}, 8.3,6.6,1.8 \mathrm{~Hz}, 1 \mathrm{H}), 7.32(\mathrm{dd}, J$ $=7.9,1.8 \mathrm{~Hz}, 1 \mathrm{H}), 7.15-7.05(\mathrm{~m}, 4 \mathrm{H}), 6.94(\mathrm{~m}, 1 \mathrm{H}), 6.45(\mathrm{~d}, J=0.8 \mathrm{~Hz}, 1 \mathrm{H}), 3.73(\mathrm{~s}, 3 \mathrm{H})$, 2.57 (m, 2H), $1.62(\mathrm{~m}, 2 \mathrm{H}), 1.36$ (sext., $J=7.4 \mathrm{~Hz}, 2 \mathrm{H}), 0.89$ (t, $J=7.3 \mathrm{~Hz}, 3 \mathrm{H}) .{ }^{13} \mathrm{C}-\mathrm{NMR}$ $\left(75 \mathrm{MHz}, \mathrm{CDCl}_{3}\right): \delta=156.2,142.7,138.2,130.5,129.6,128.2,126.5,120.8,120.7,119.6$, $119.5,112.3,109.8,99.3,55.6,30.5,26.4,22.3,13.8$. MS (EI) $m / z$ (relative intensity) 279 (90) $\left[\mathrm{M}^{+}\right], 237$ (100), 222 (99), 204 (33). HR-MS (EI) m/z calcd for $\mathrm{C}_{19} \mathrm{H}_{21} \mathrm{NO} 279.1623$, found 279.1605 . 
12.0

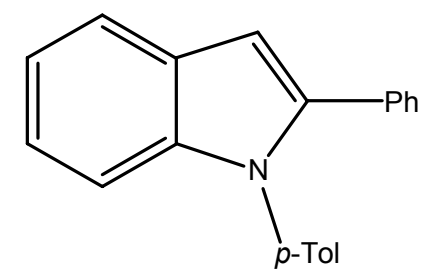

$4 a$

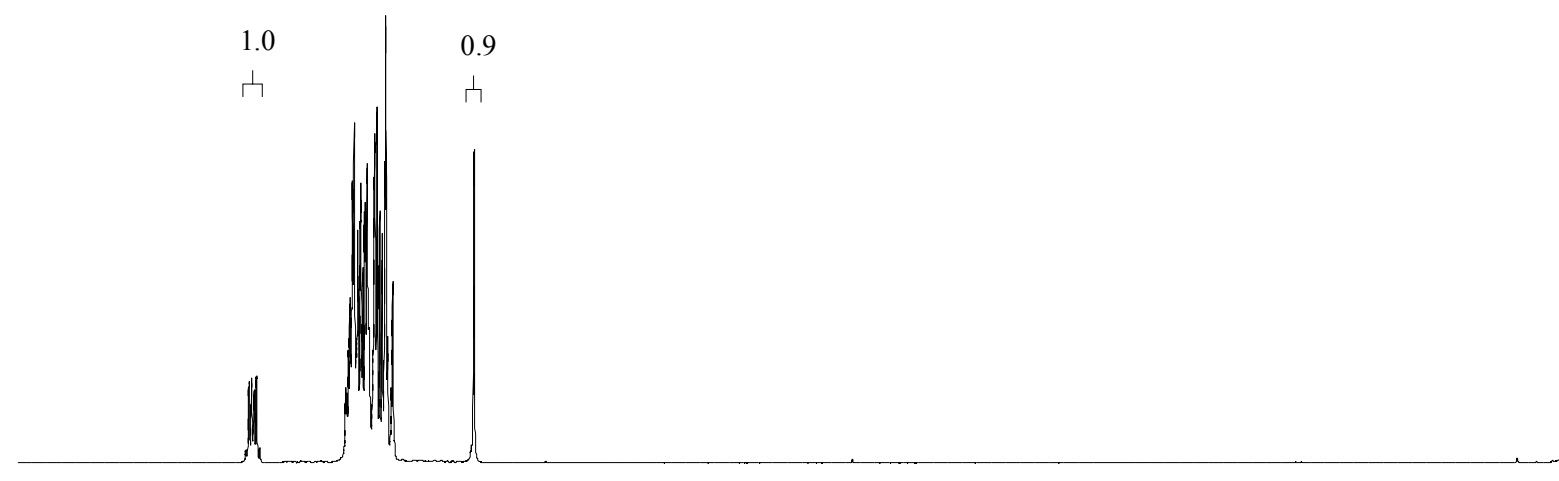

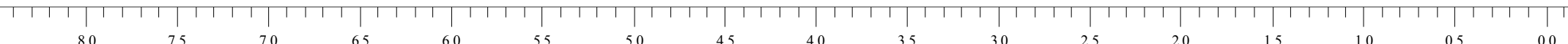




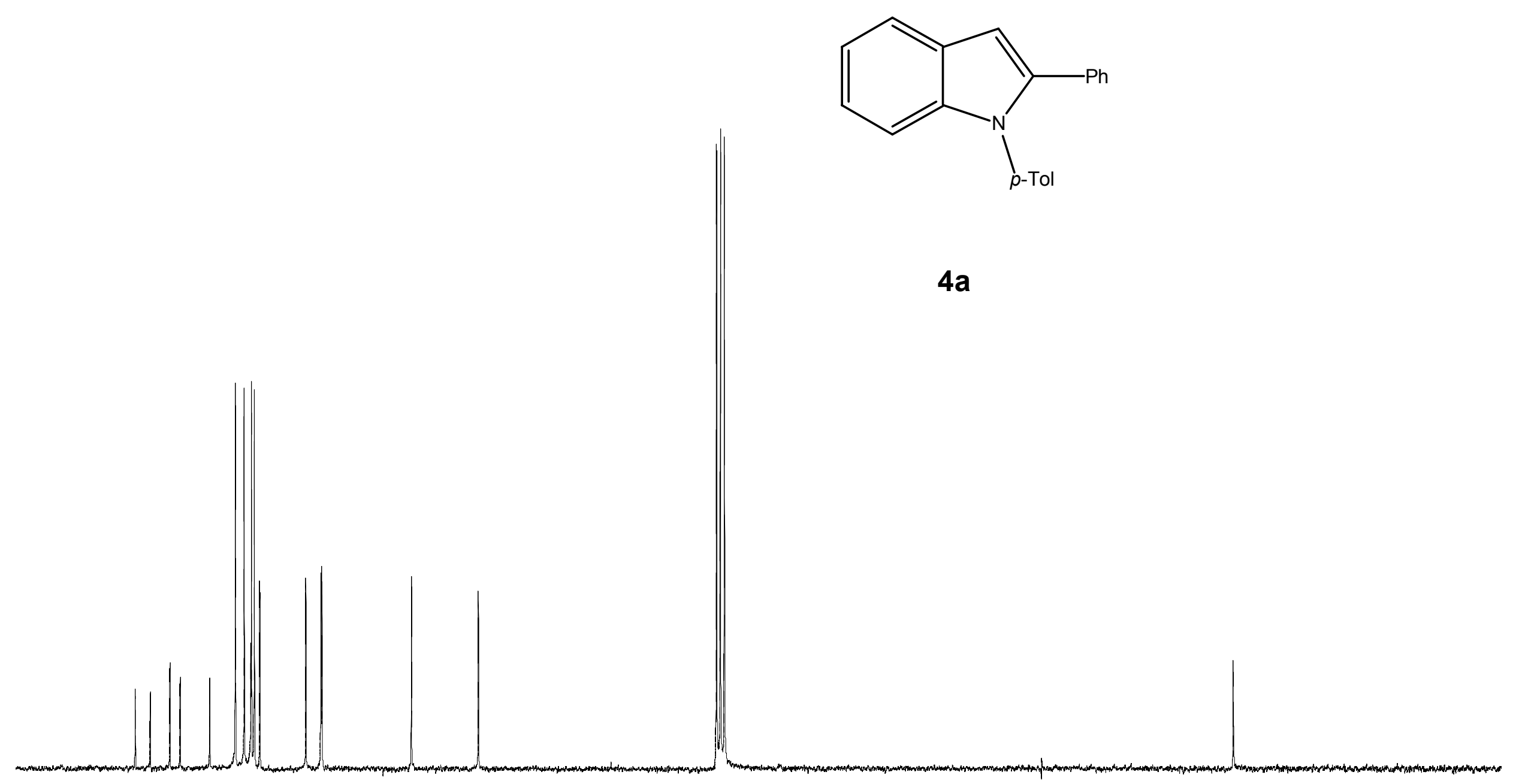

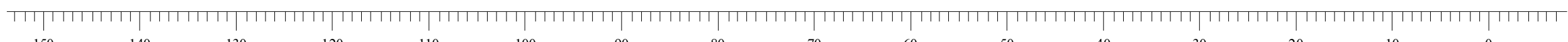



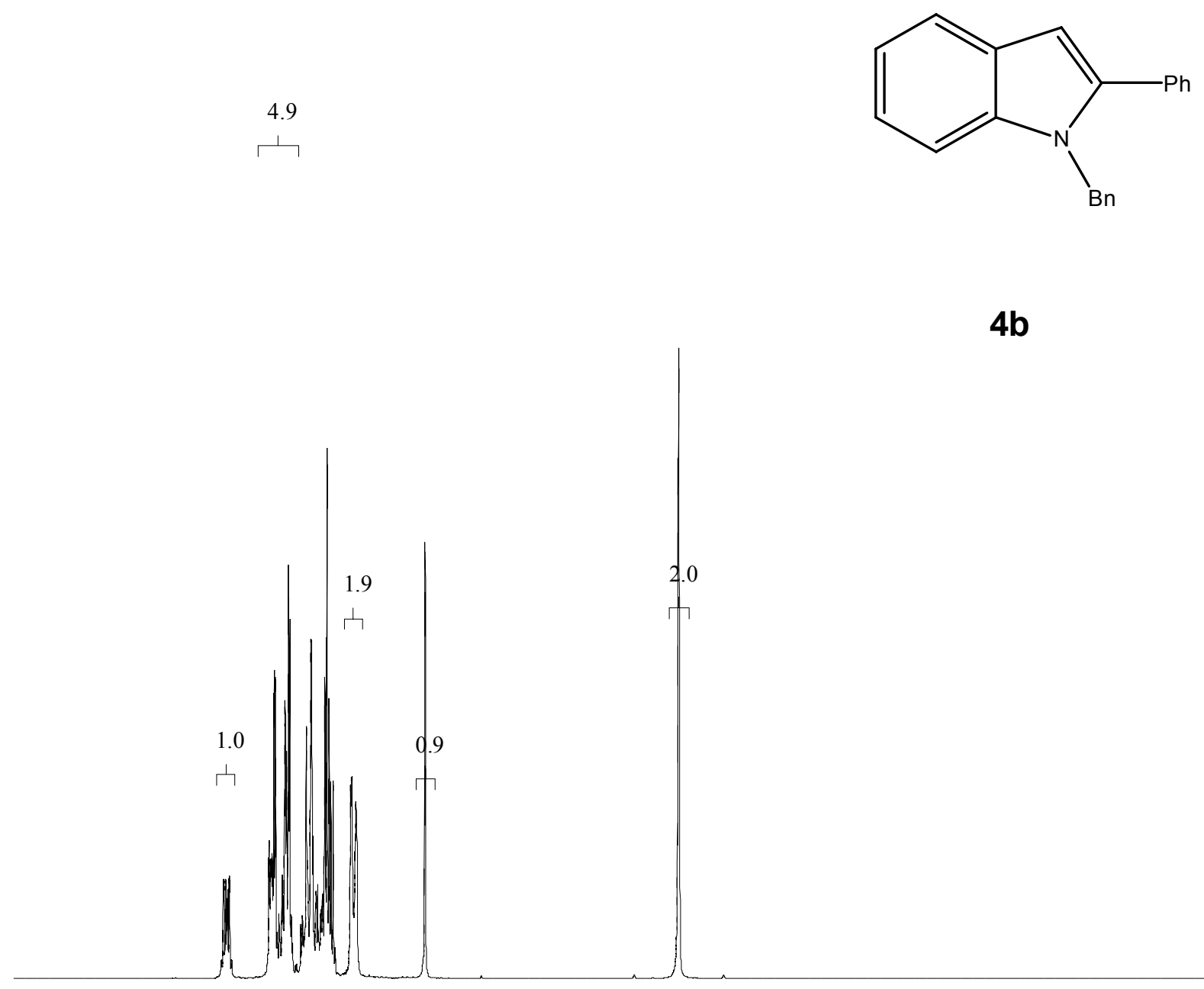

b

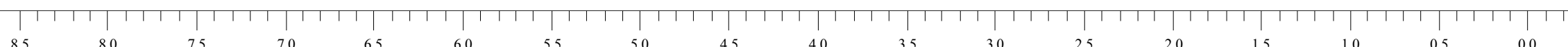




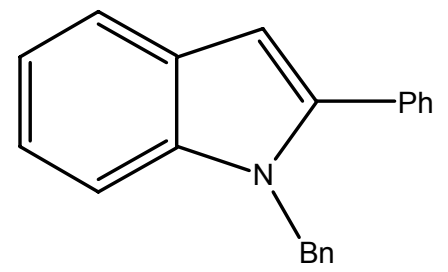

4b
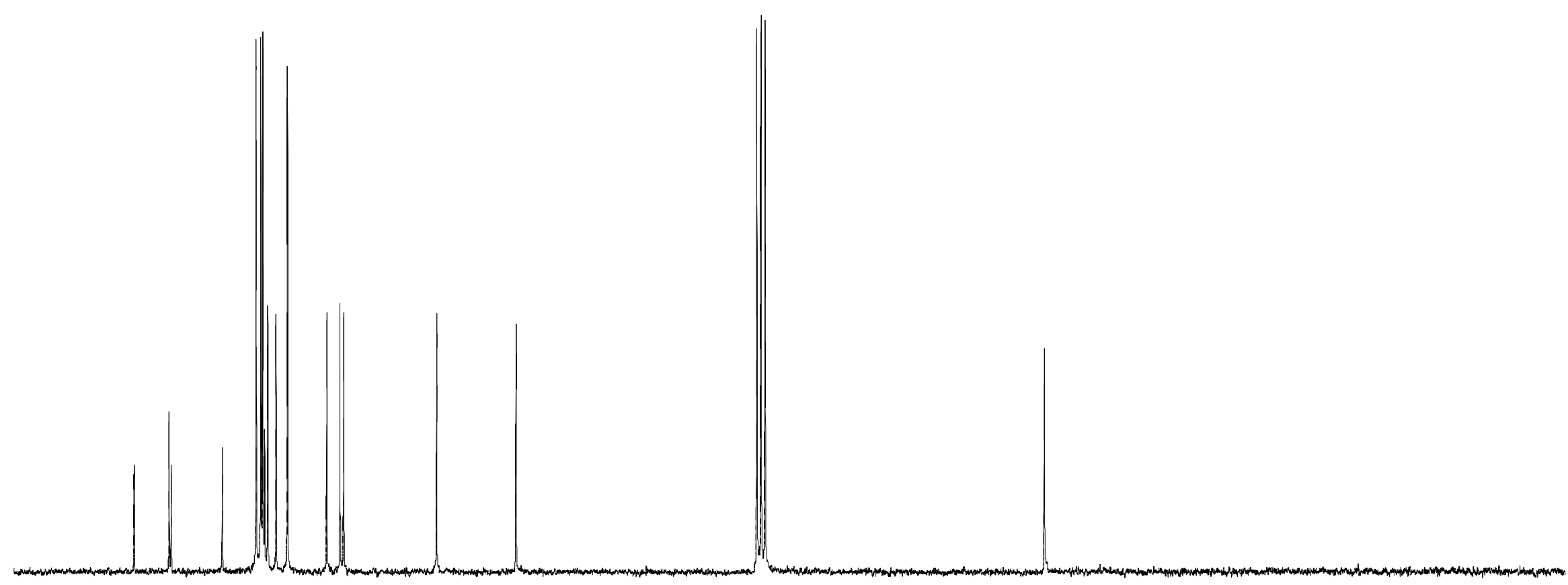

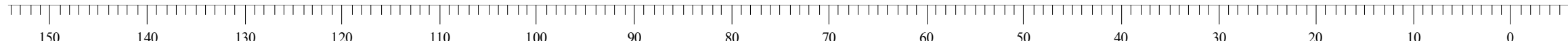




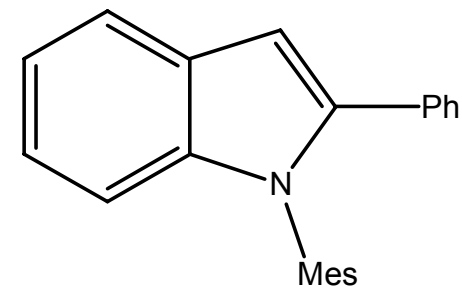

1.0

i

2.0

ウ

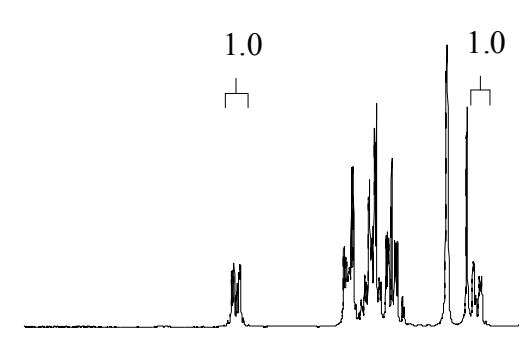

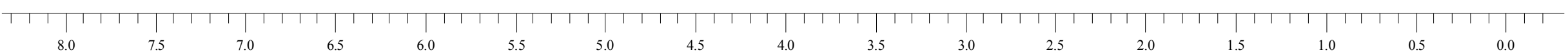




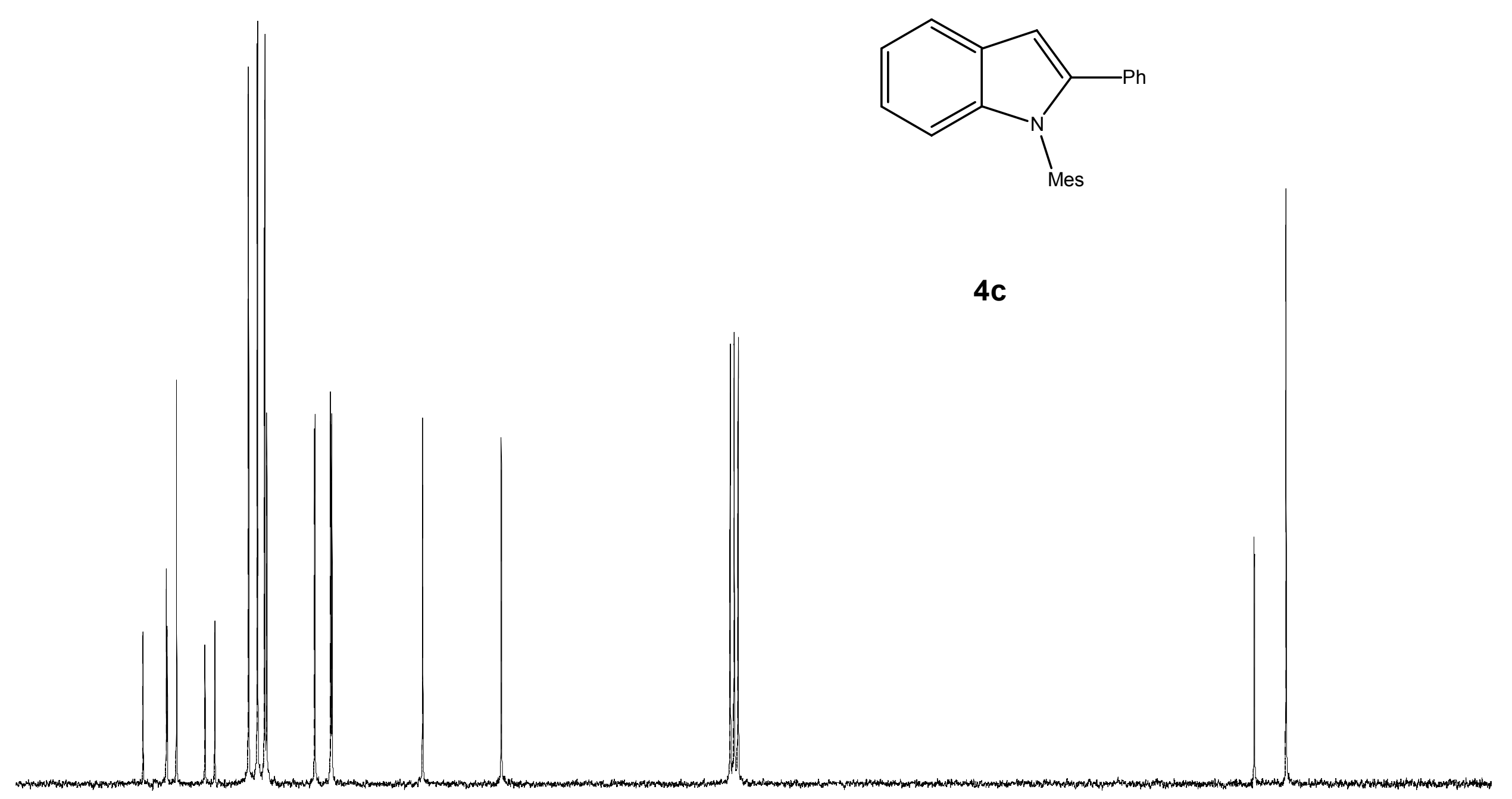

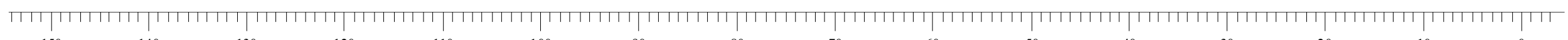

$140 \quad 130$

120

110

80

70

$60 \quad 50$

40

30

20

$10 \quad 0$ 


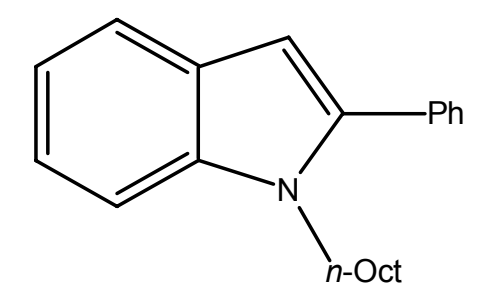

10.3

4d

6.0

$\vdash 1.2$

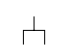

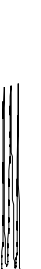




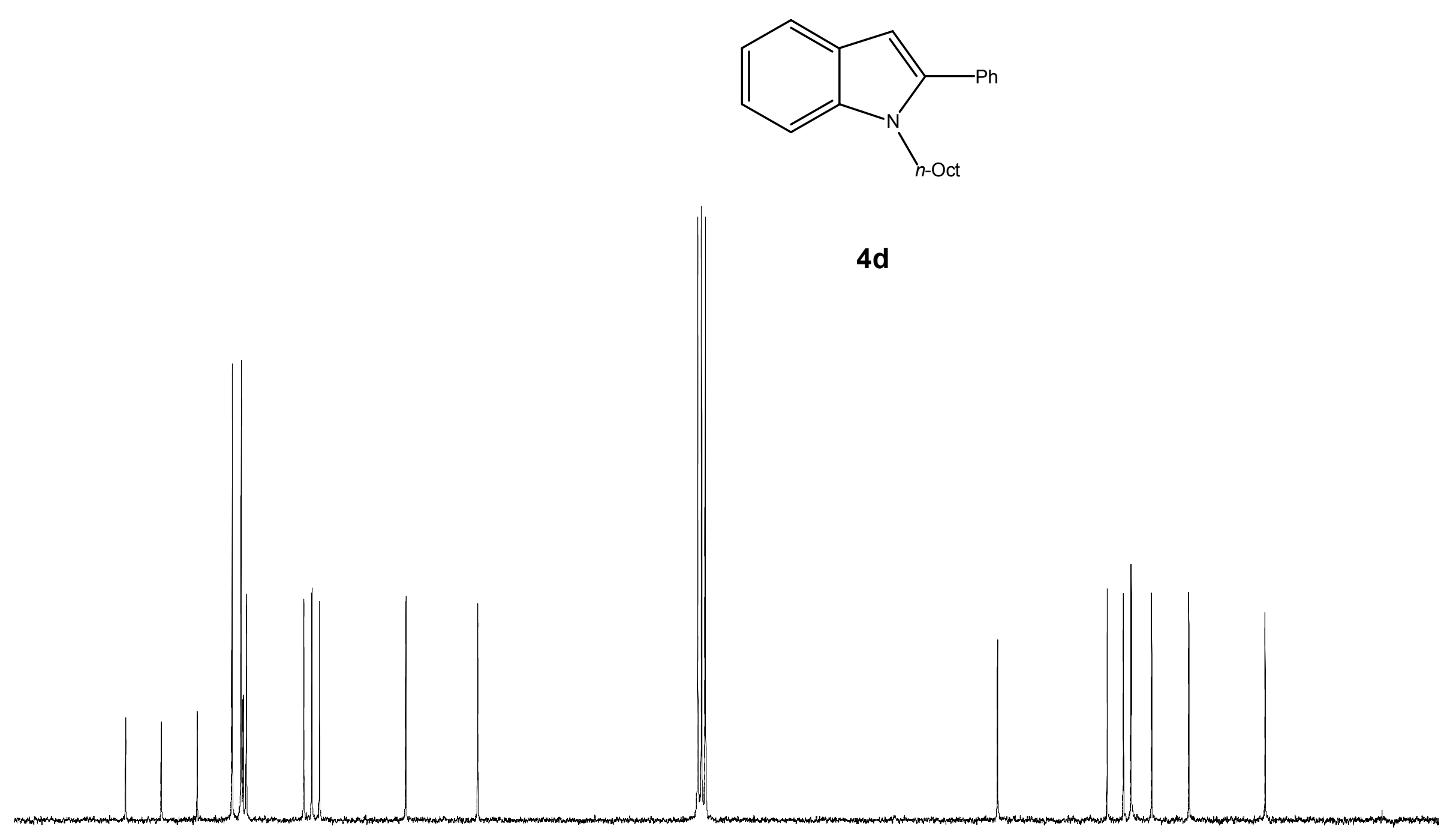


(1)

4 e

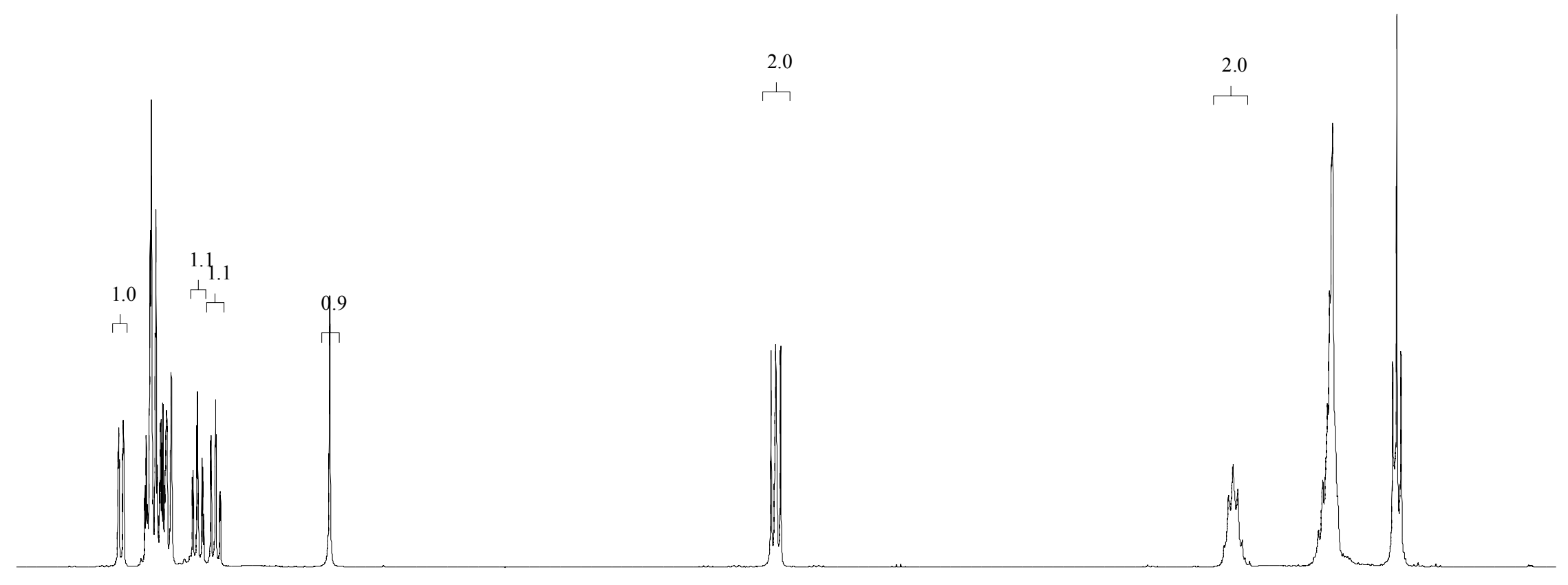

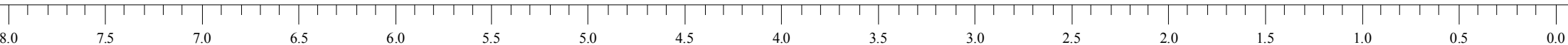




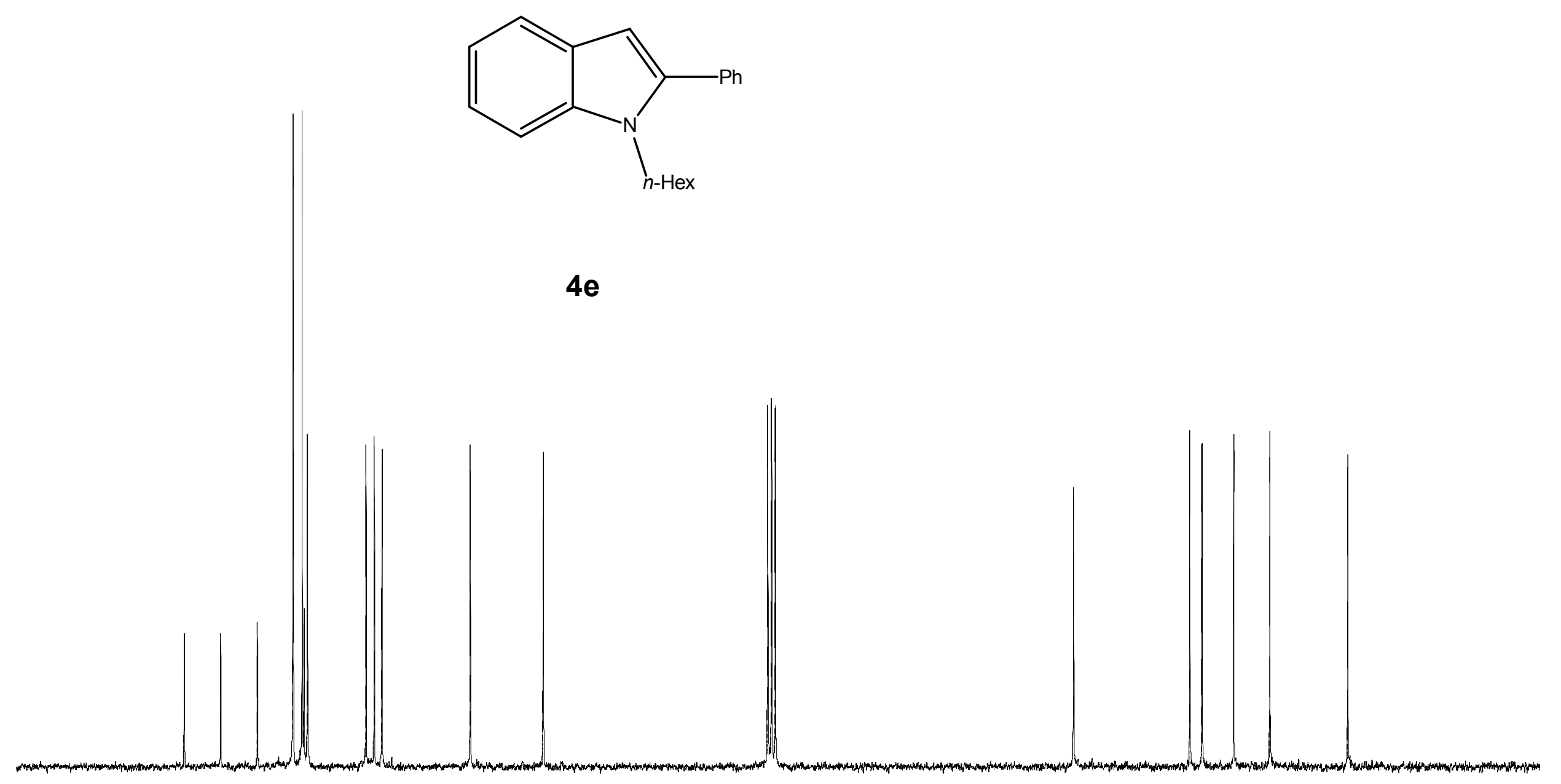

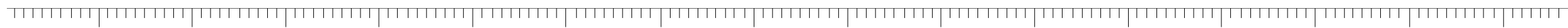


4.9

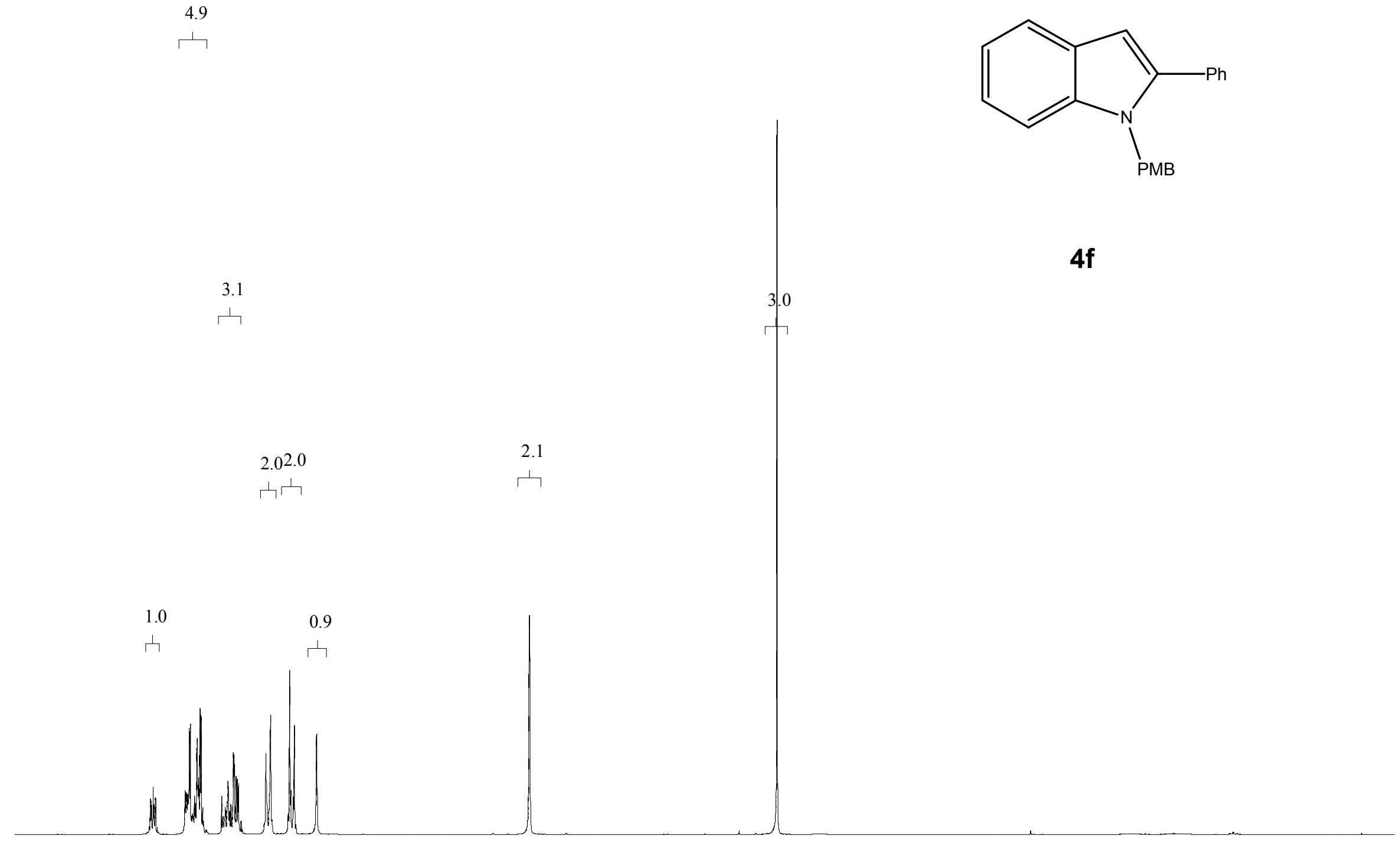

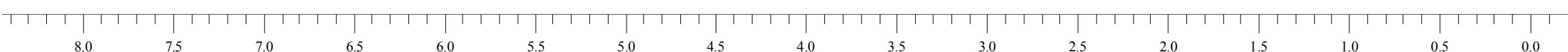




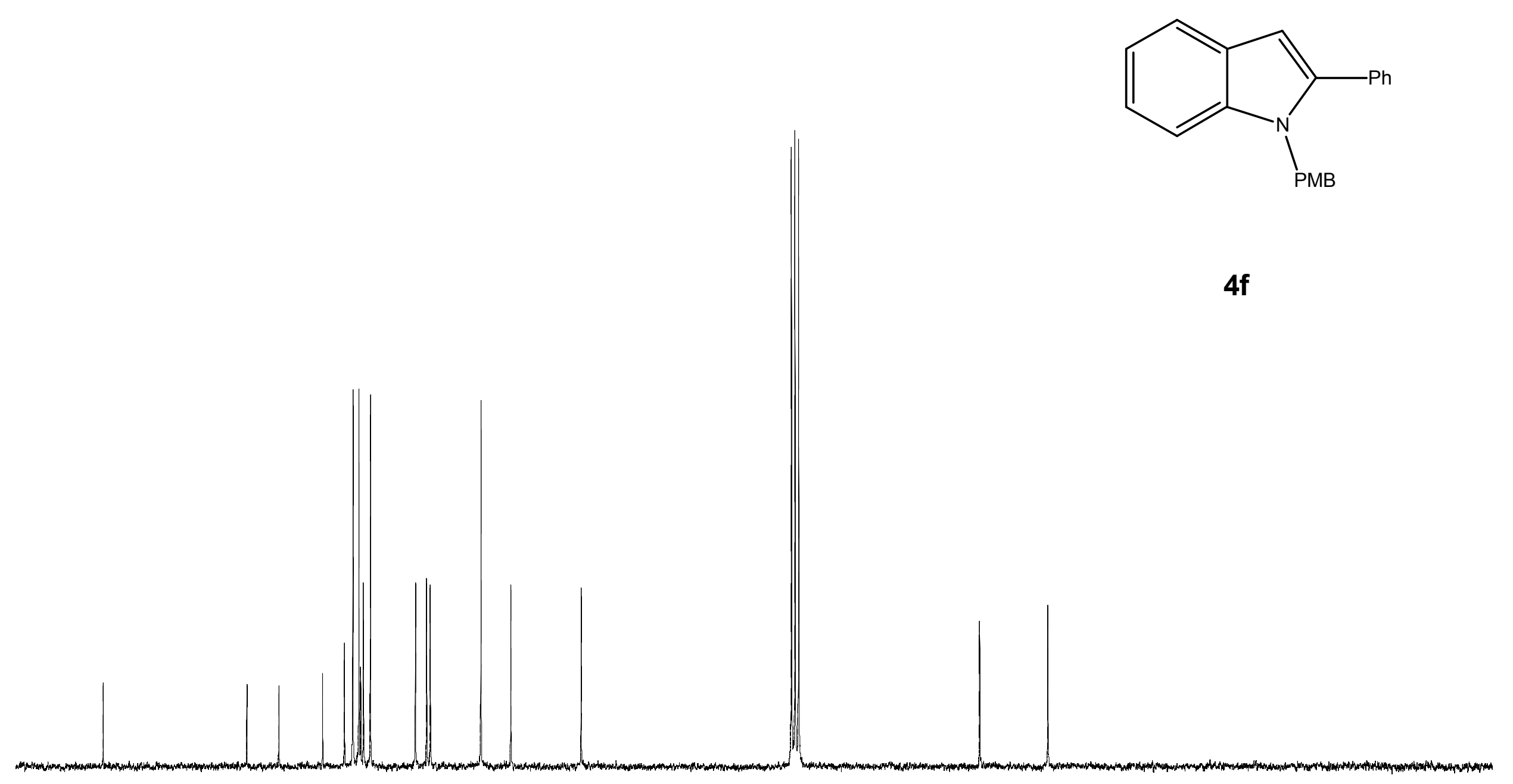




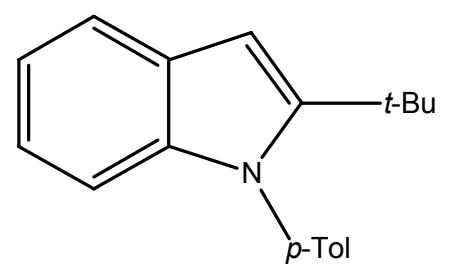

$4 g$

3.0

2.0

$1.0 \quad 0.91 .0$

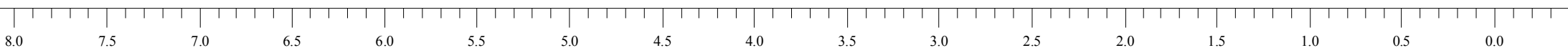




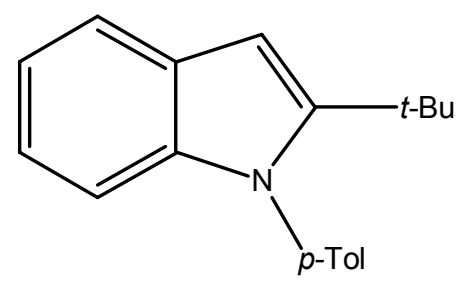

\section{$4 g$}
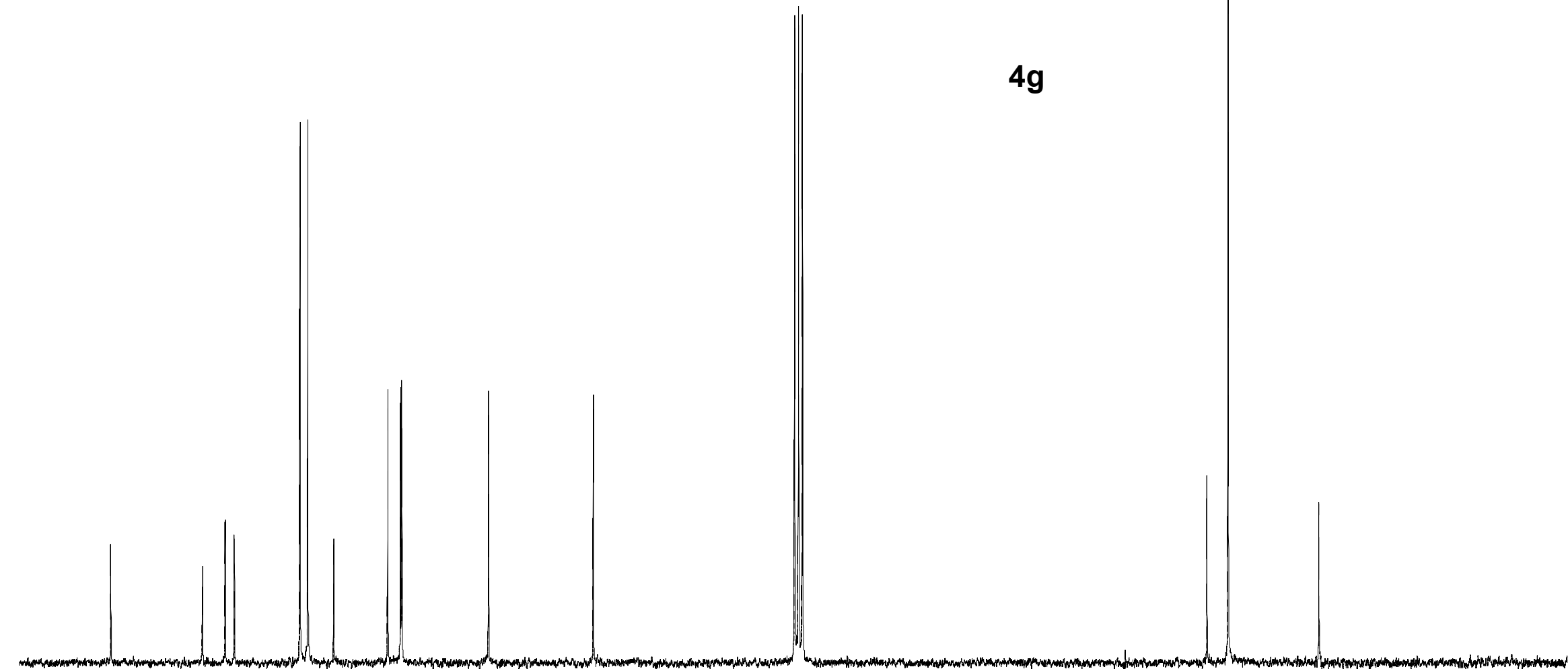

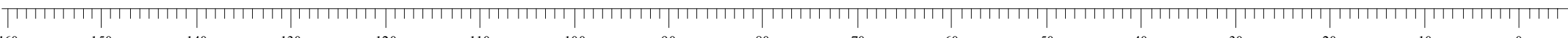




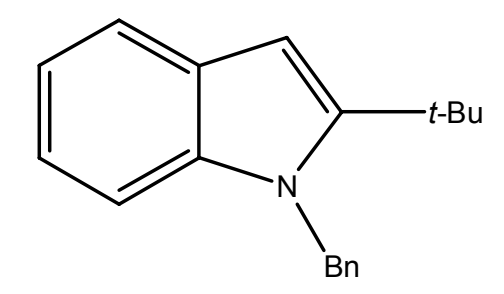

9.0

\section{4h}

3.1

3.0

$1.9 \quad 2.0$

$0.9 \quad 1.0$

内 ウ

H_...Hith 

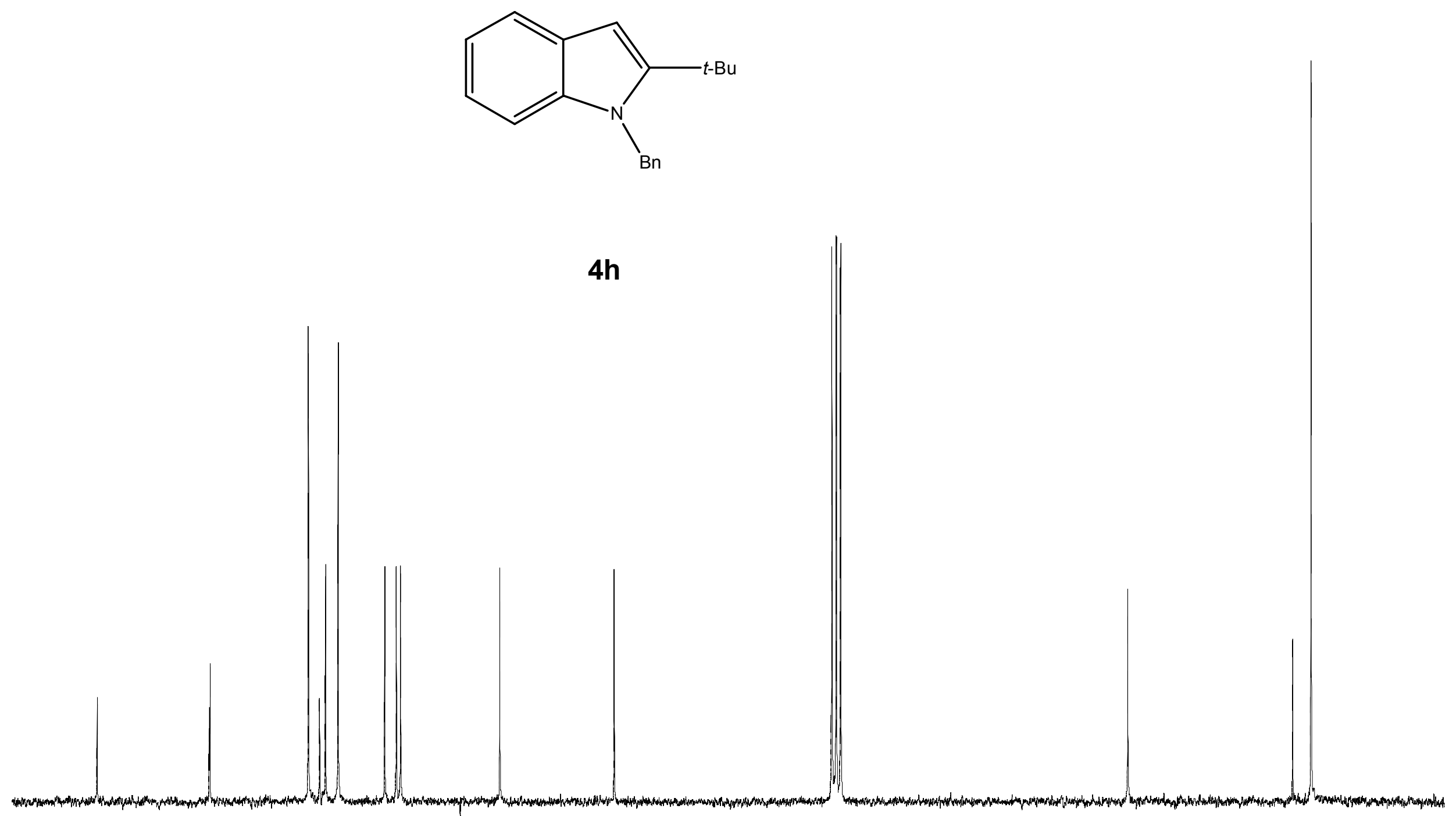

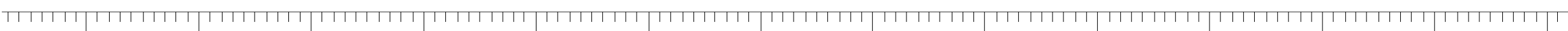


<smiles>CCOC(=O)c1ccc(-n2c(OCC)cc3cc(C(F)(F)F)ccc32)cc1</smiles>

1.9

2.0

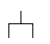

ウ

2.0

4j
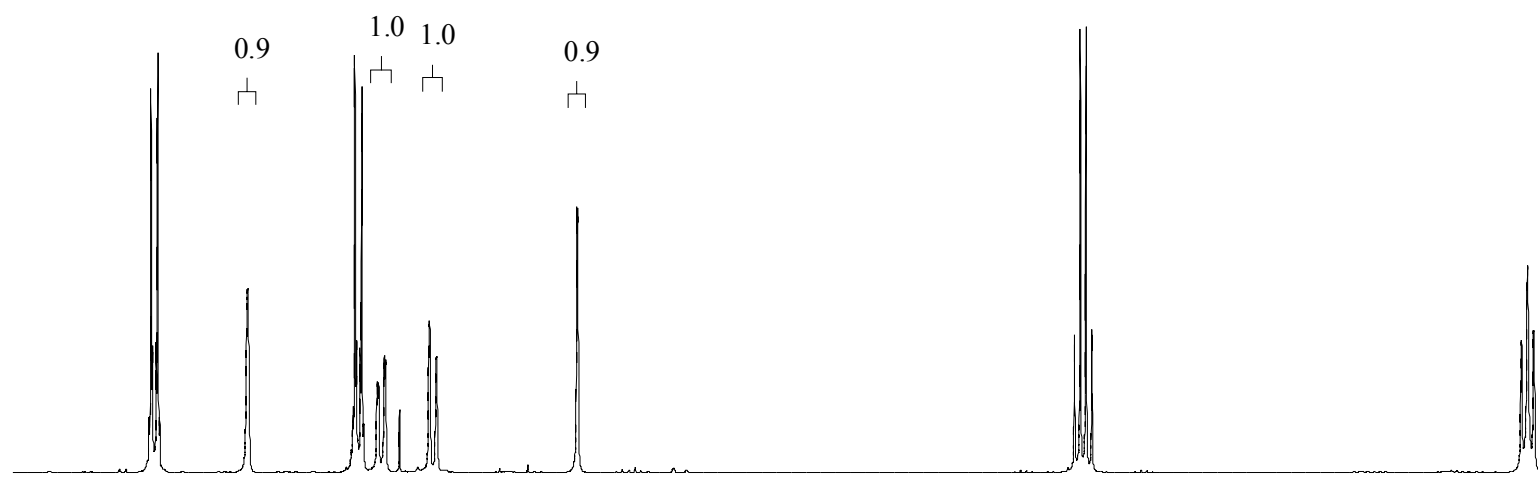

1.9

3.1

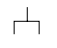

2.9

म

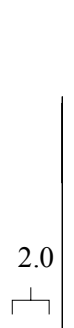

$\mapsto$

6.0

$\vdash$

$\sqcap$
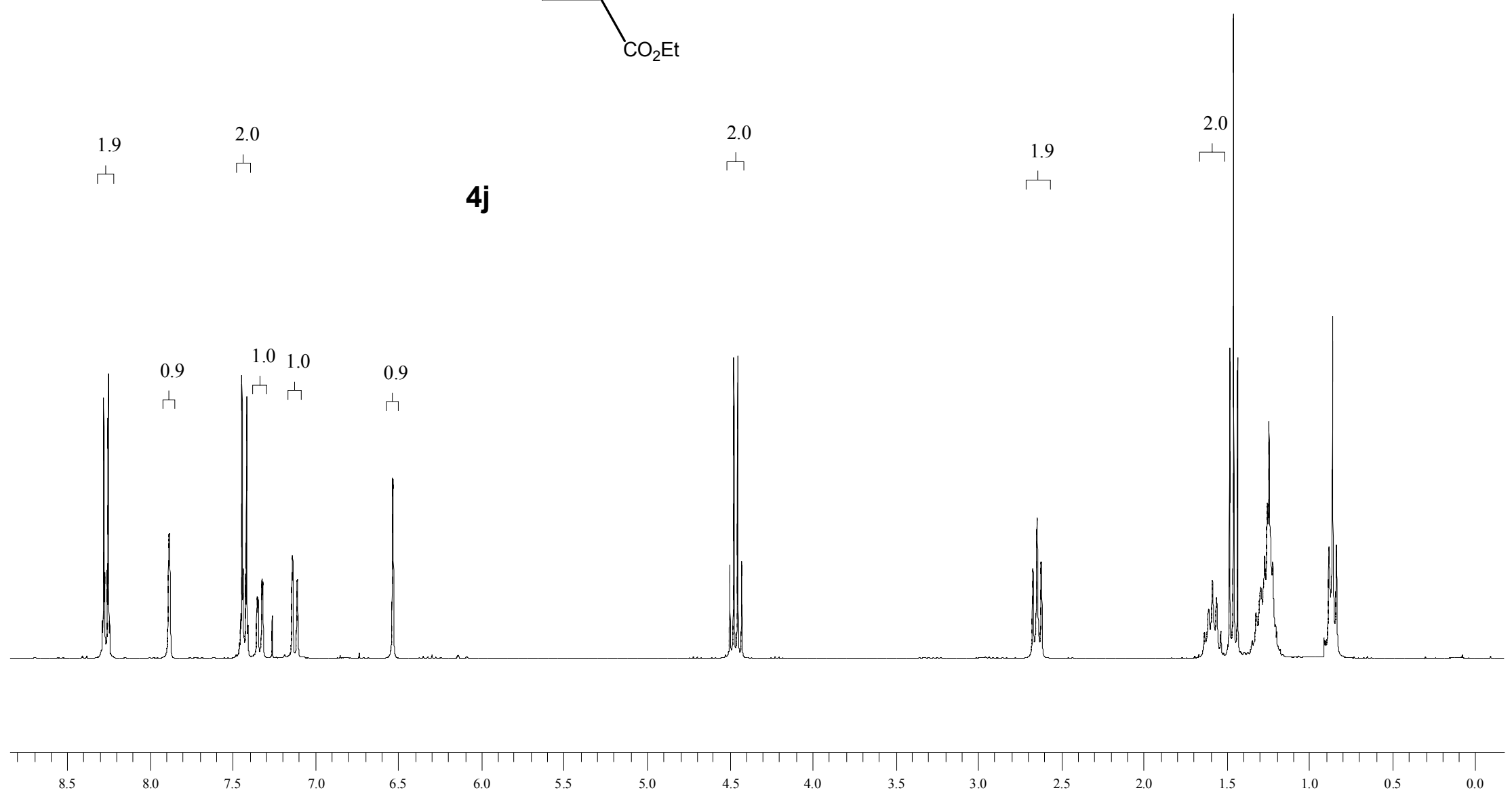

28 


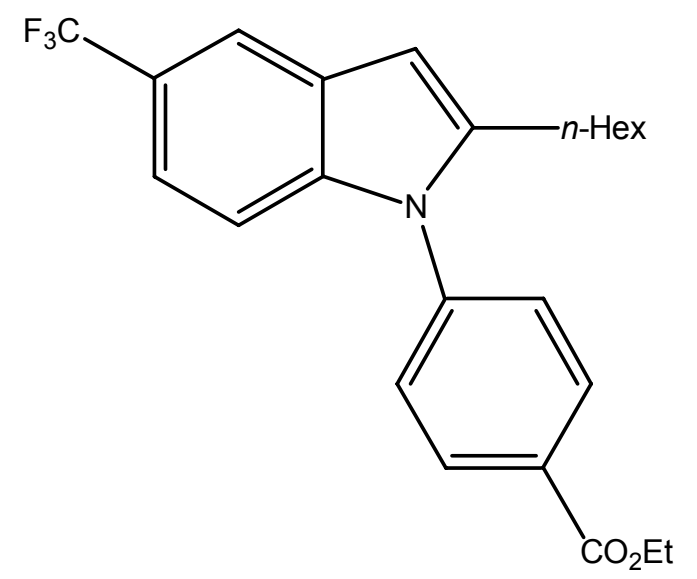

4j

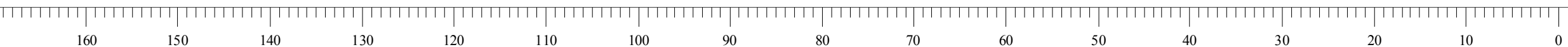
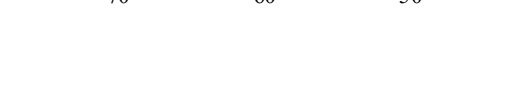


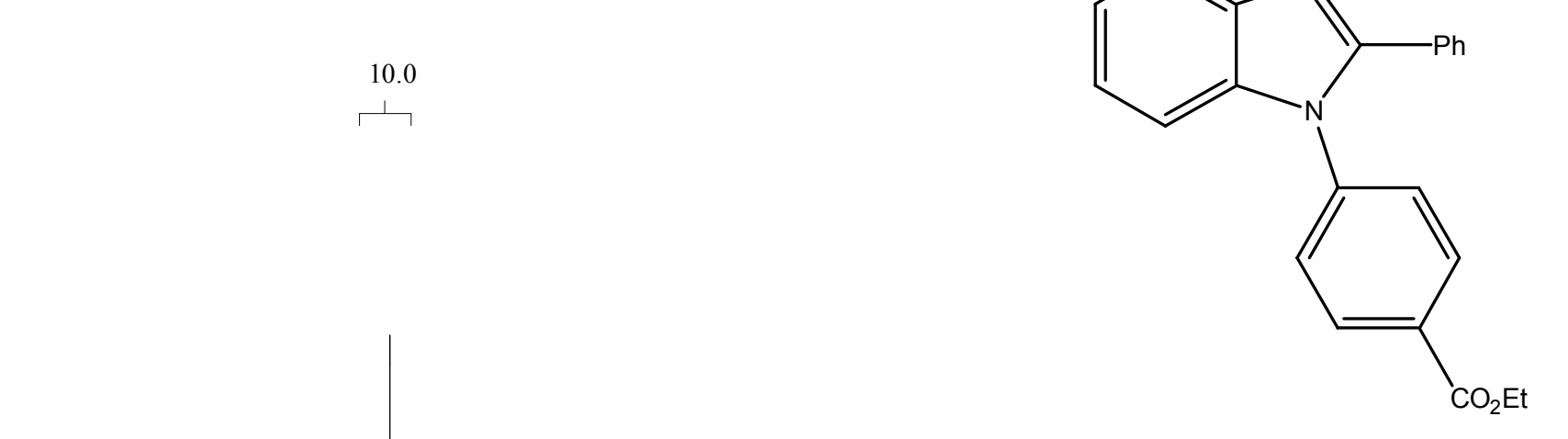

$4 k$

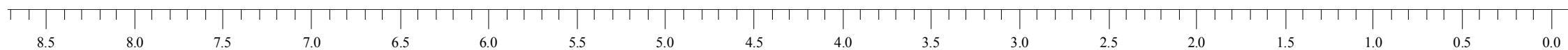




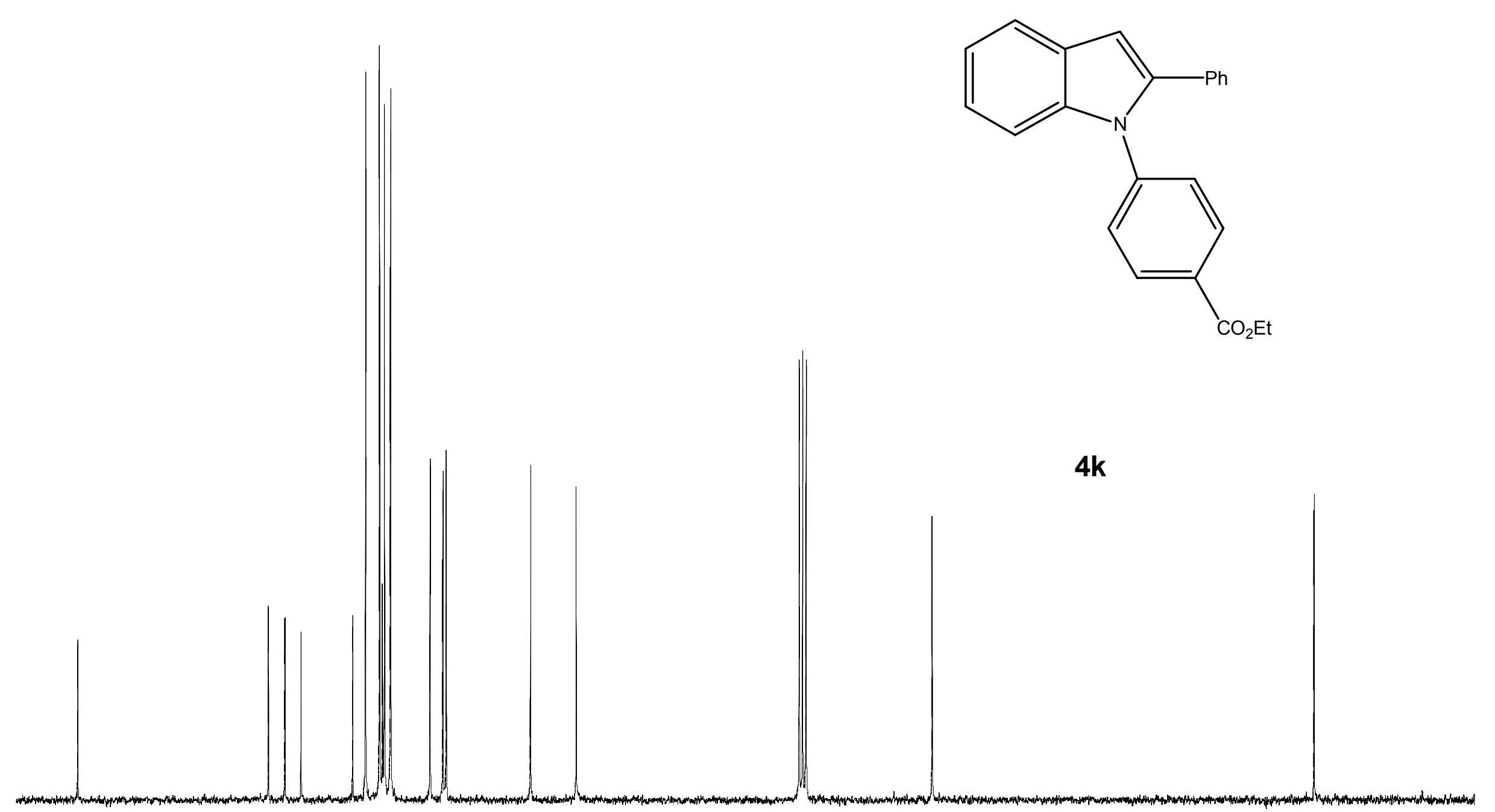

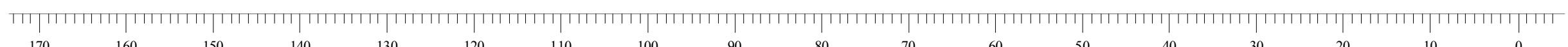




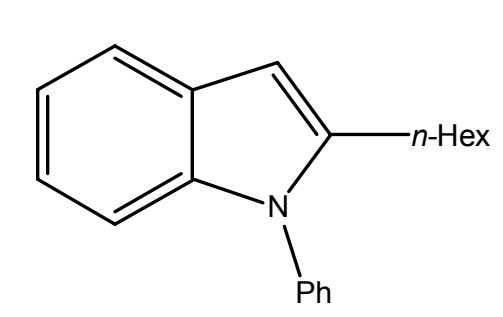

\section{$4 m$}

3.9

3.0

$\sqcap$

1.9

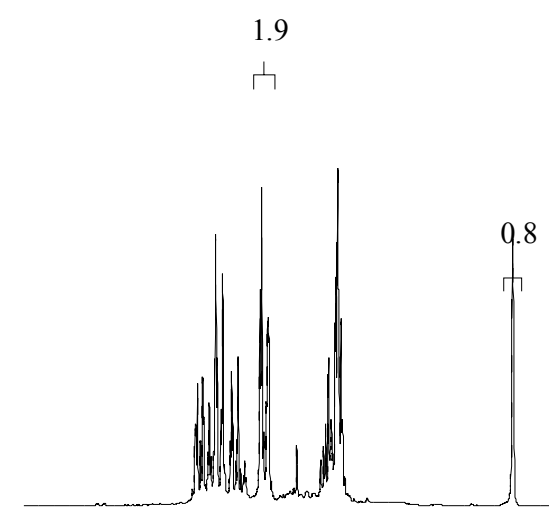

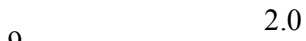

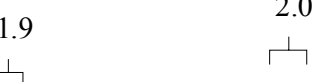



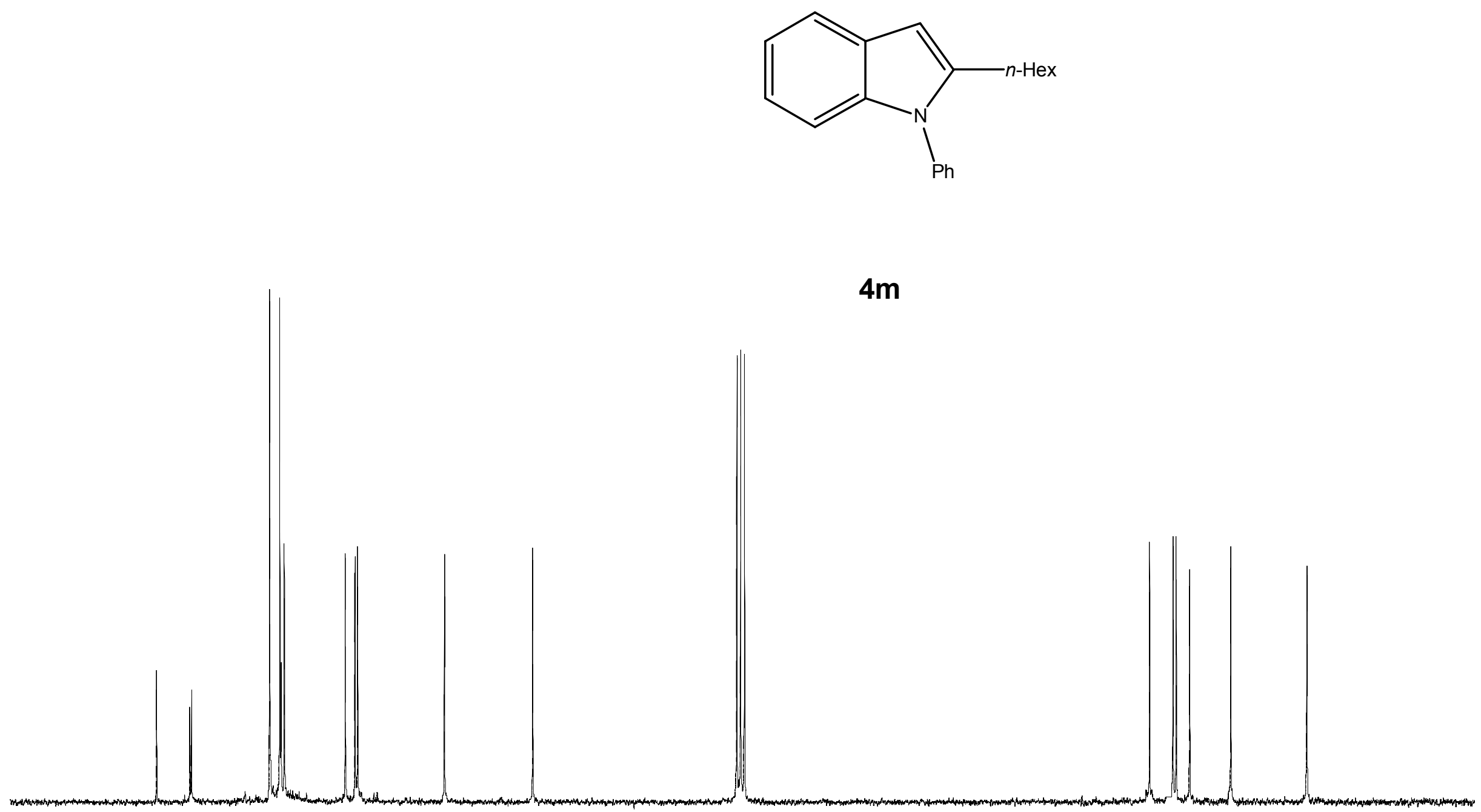

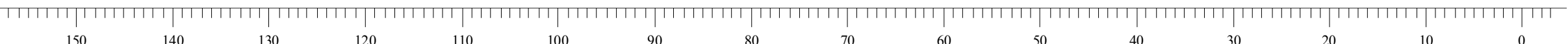




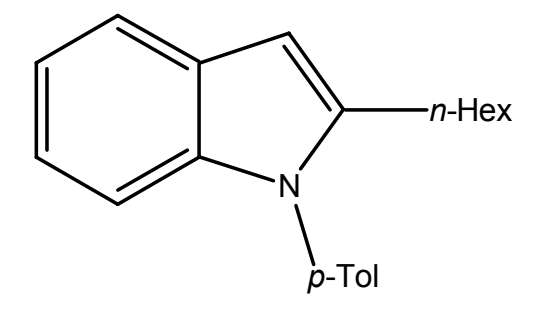

$\sqcap$

\section{«n}
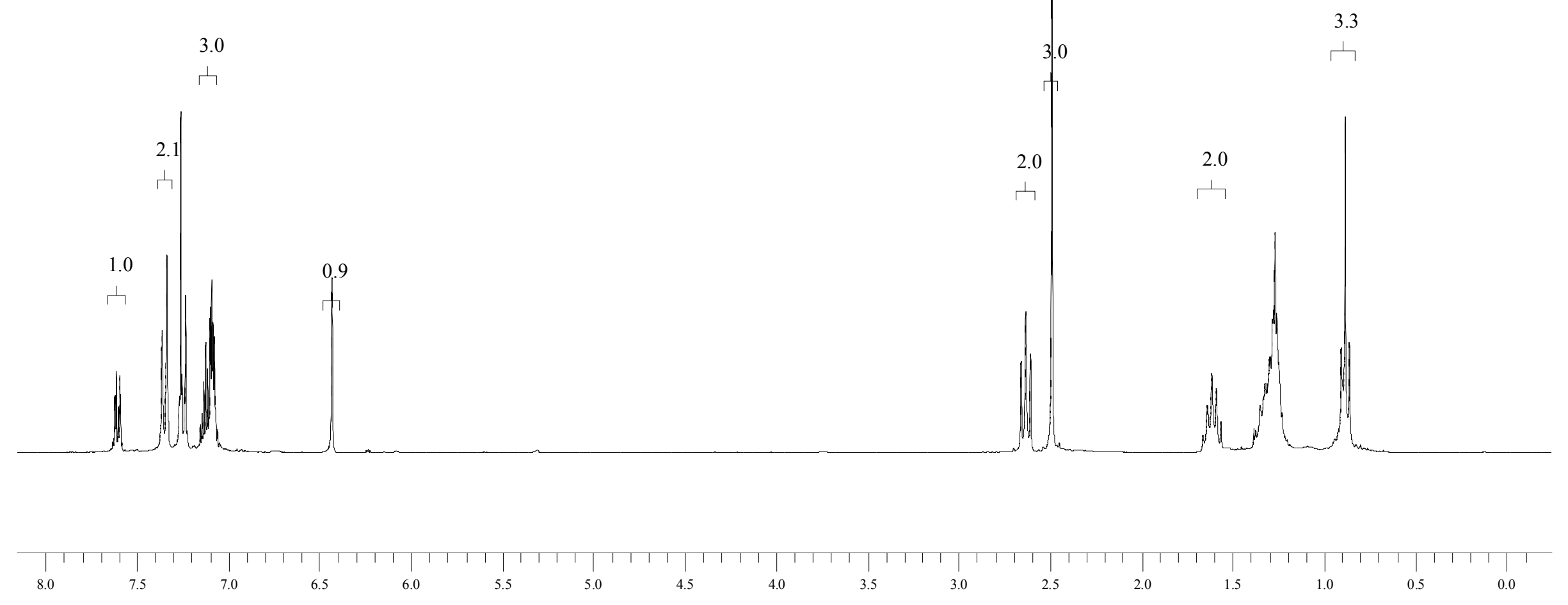


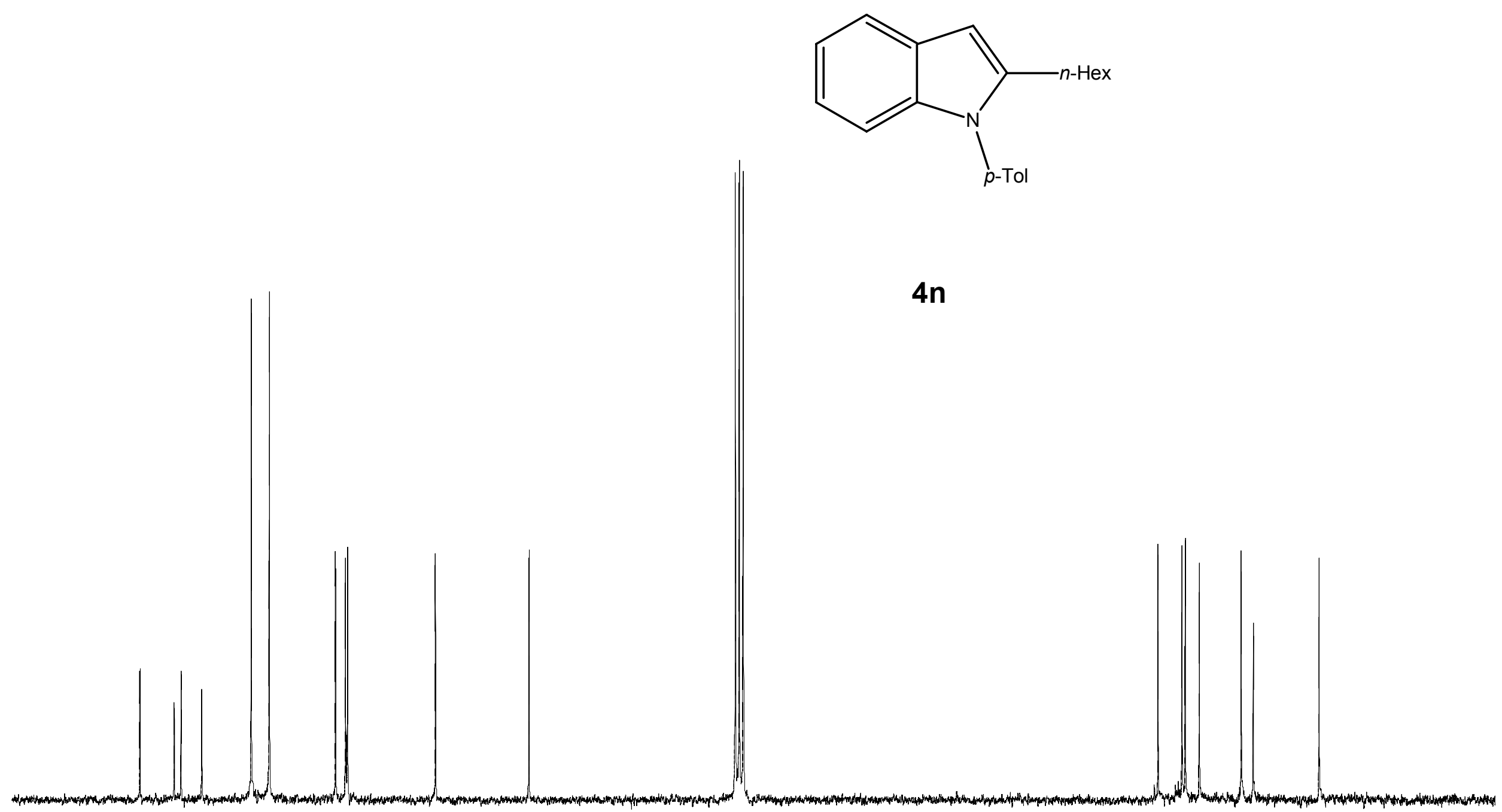

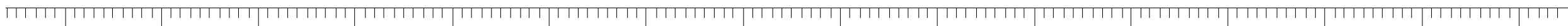


1.9

$\sqcap \quad 1.8$

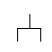

4o

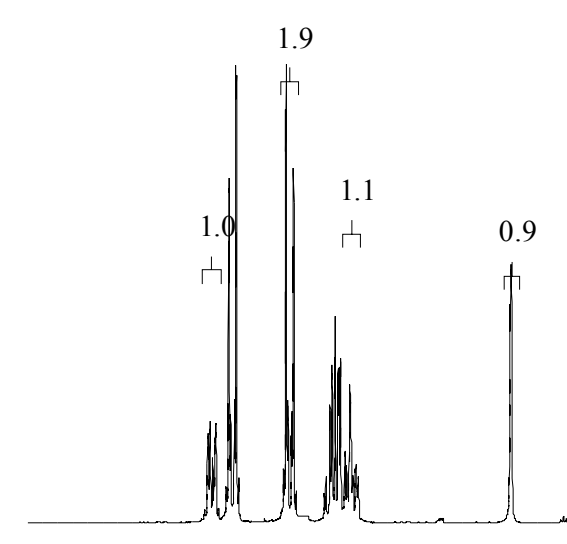

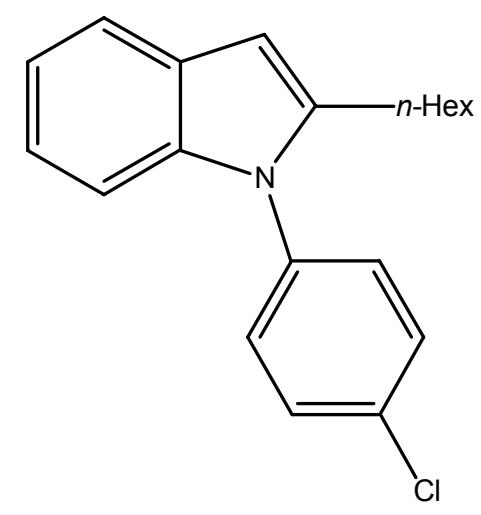
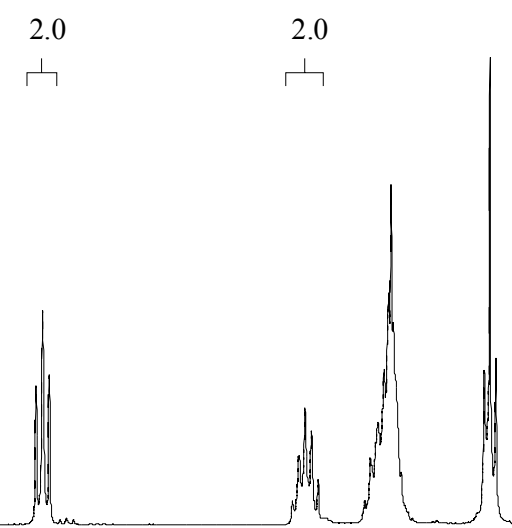


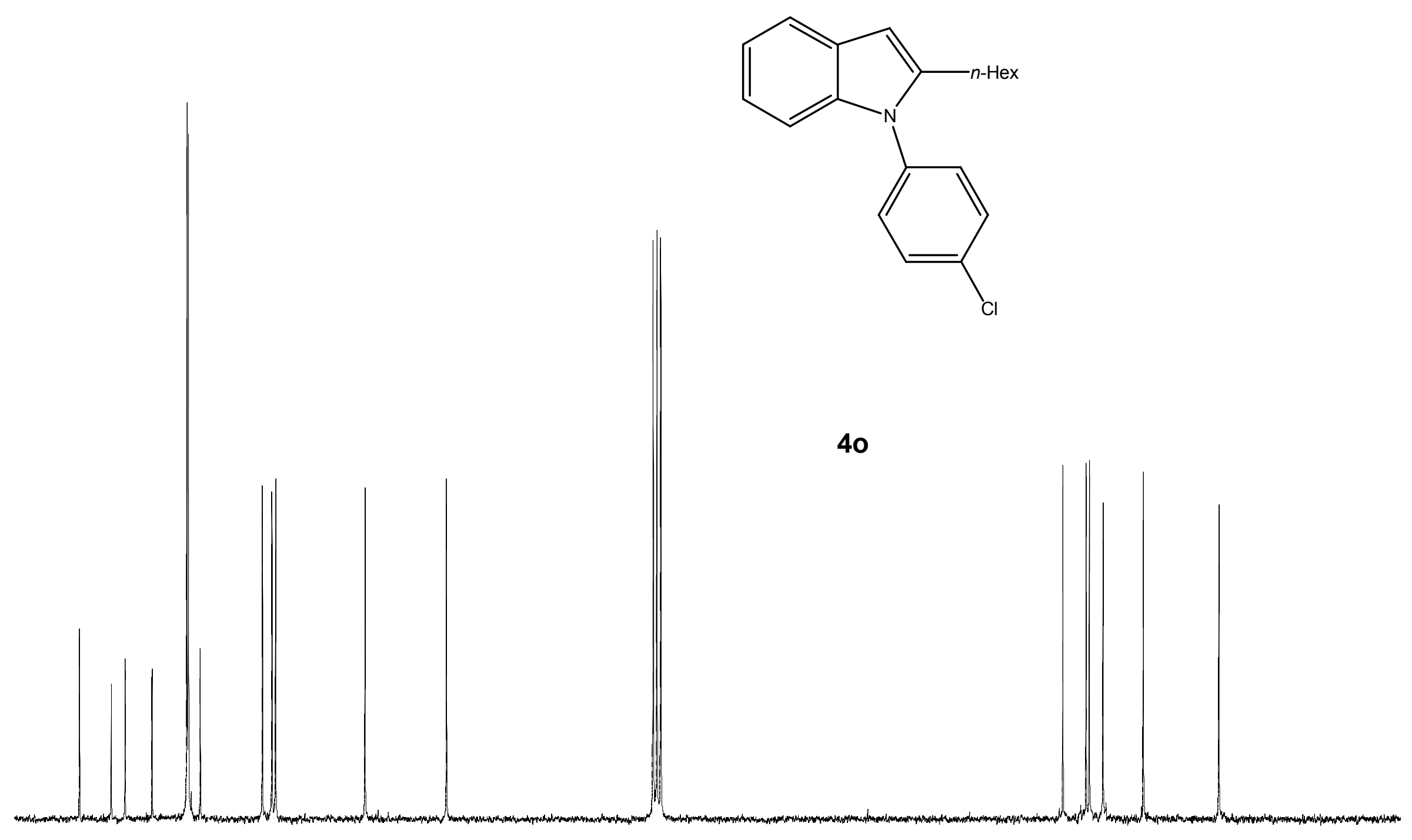

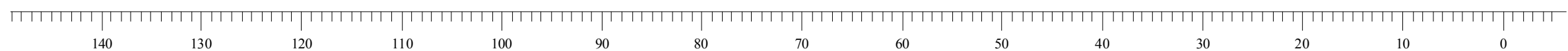



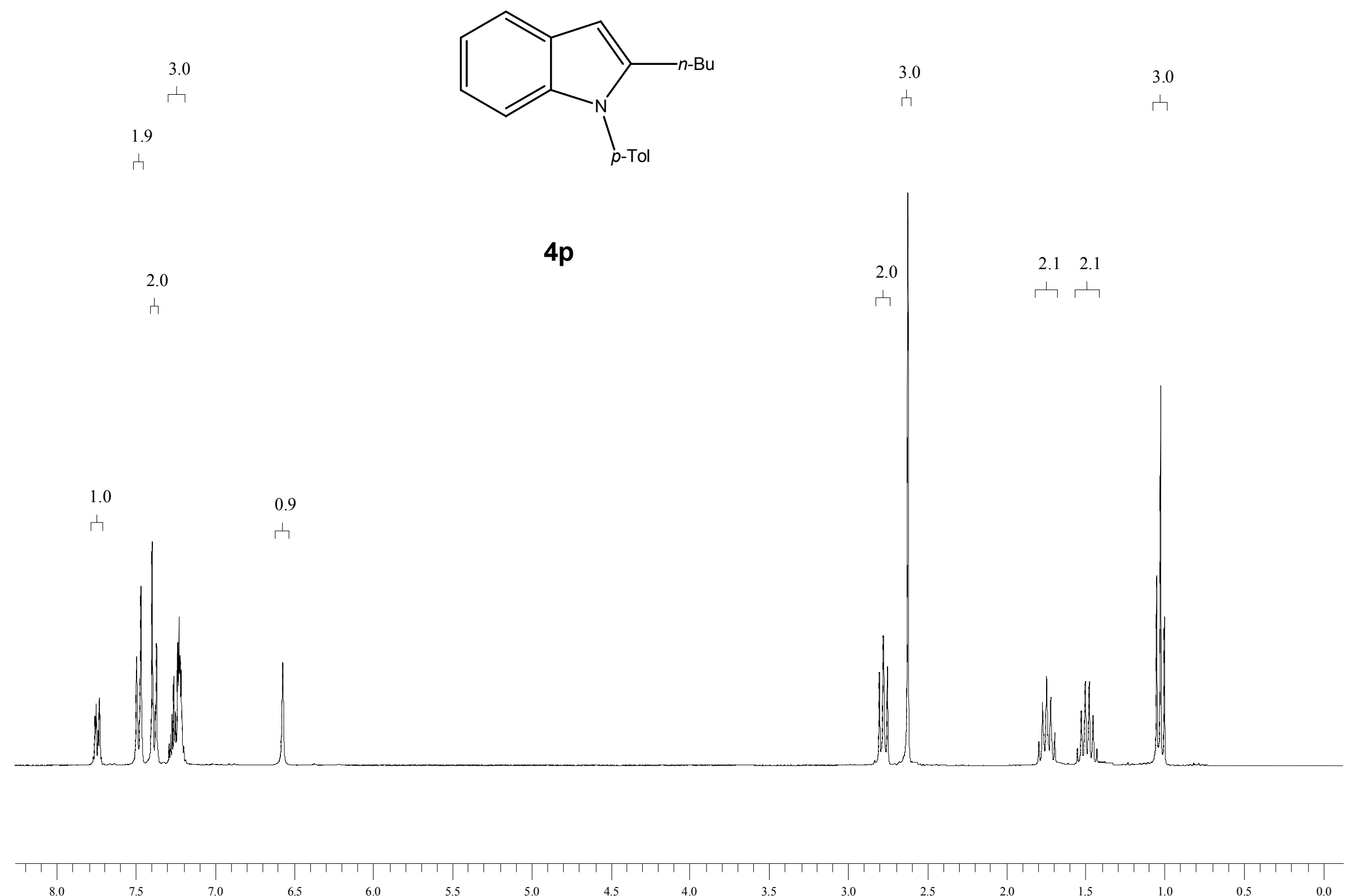


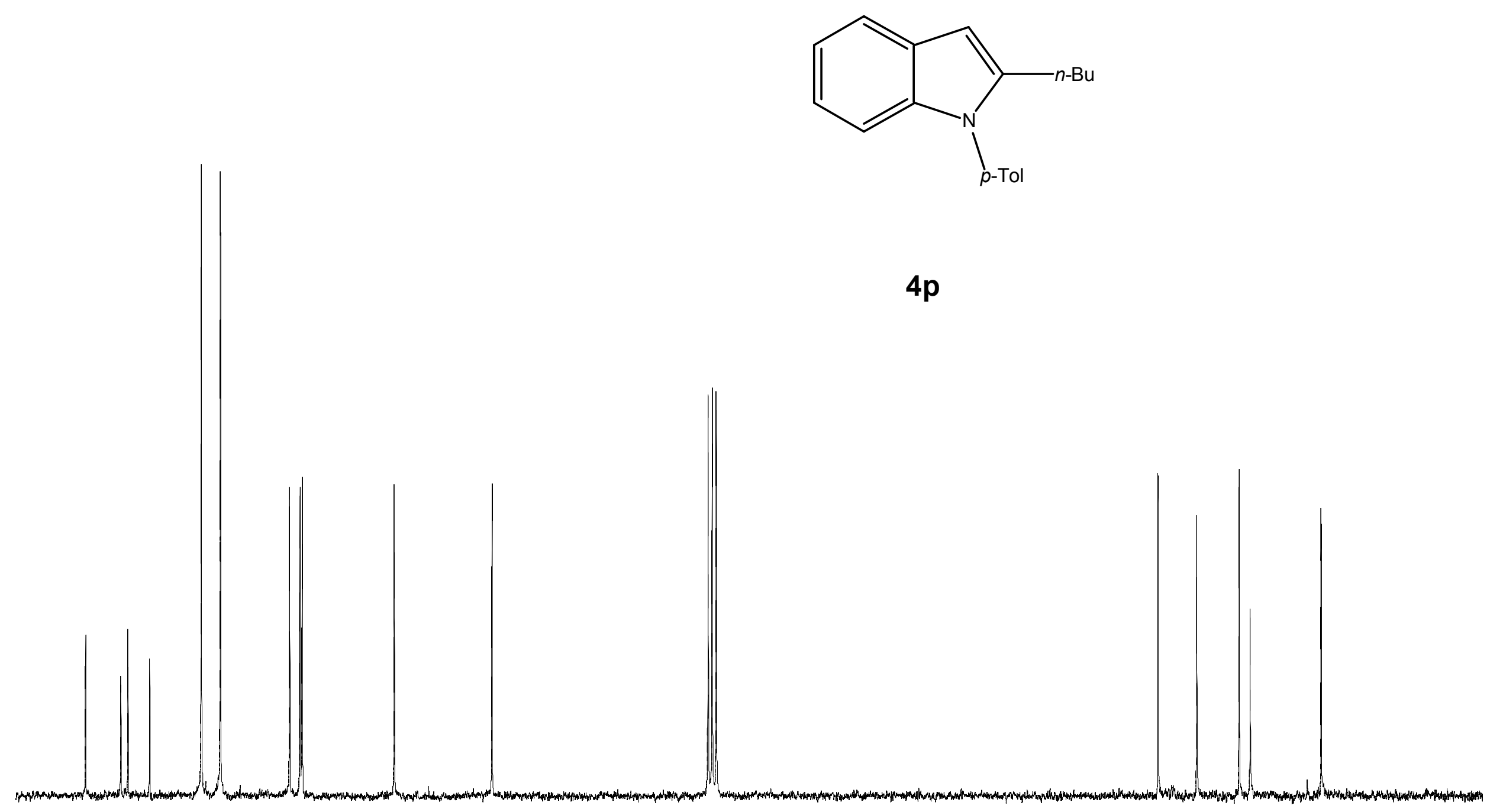

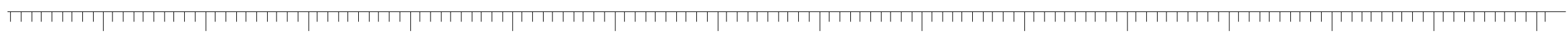



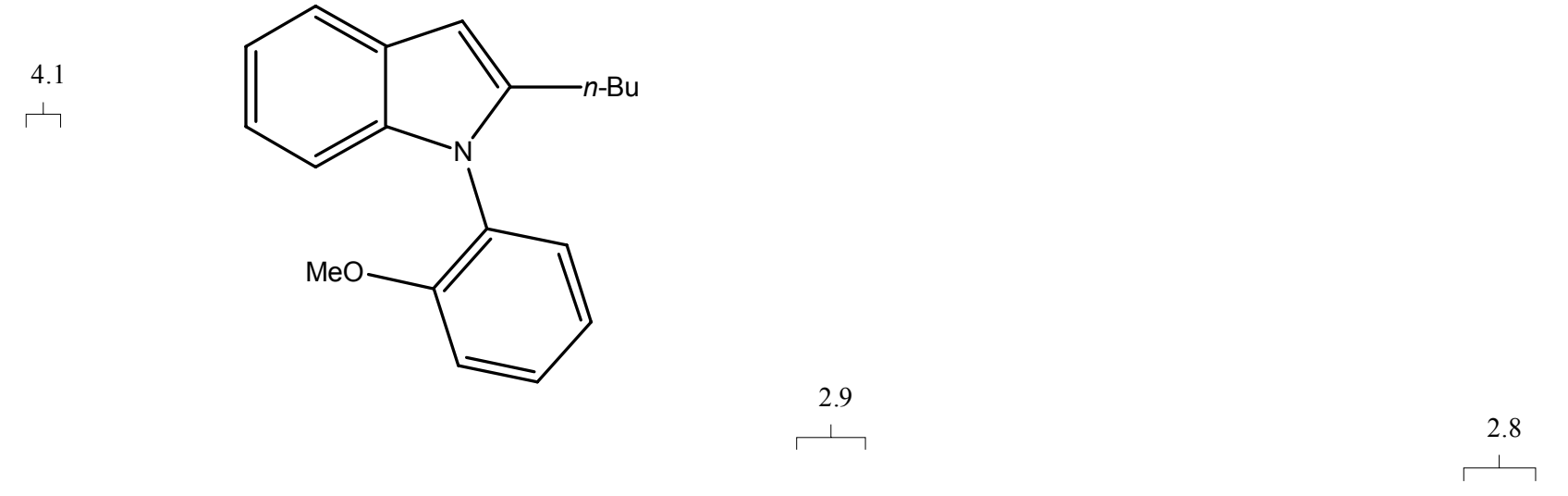

\section{$4 q$}

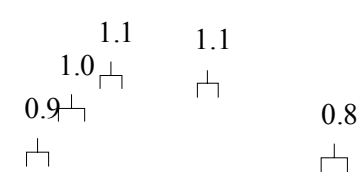

Whith 

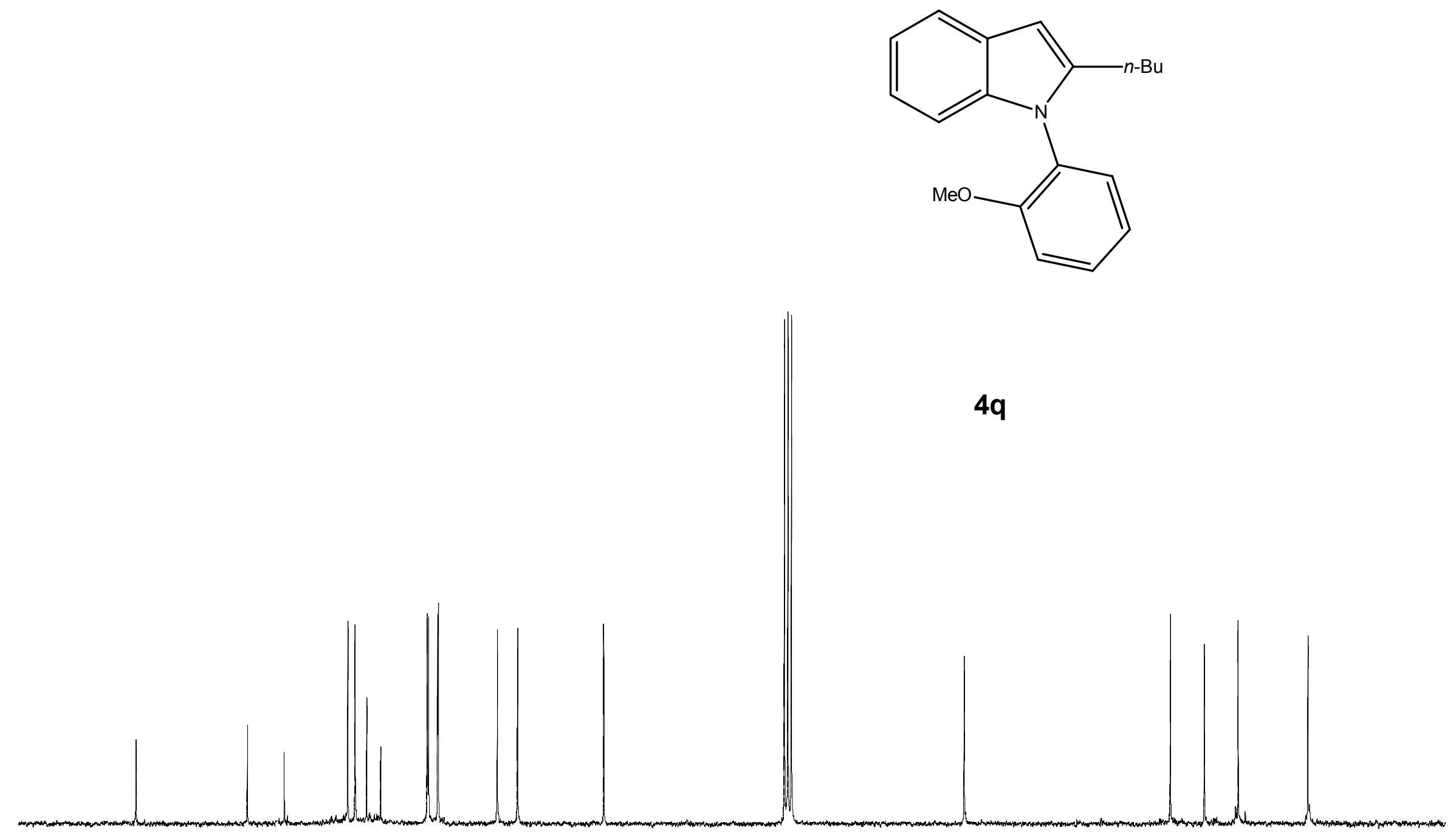

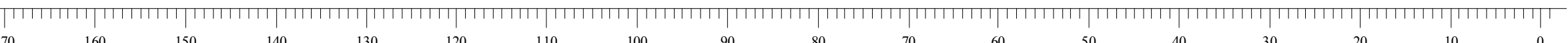

